\title{
Neutrino mixing and masses in SO(10) GUTs with hidden sector and flavor symmetries
}

\author{
Xiaoyong $\mathrm{Chu}^{a}$ and Alexei Yu. Smirnov ${ }^{b, a}$ \\ ${ }^{a}$ International Centre for Theoretical Physics, \\ Strada Costiera 11, I-34100 Trieste, Italy \\ ${ }^{b}$ Max-Planck-Institute for Nuclear Physics, \\ Saupfercheckweg 1, D-69117 Heidelberg, Germany \\ E-mail: xchu@ictp.it, smirnov@mpi-hd.mpg.de
}

ABSTRACT: We consider the neutrino masses and mixing in the framework of $\mathrm{SO}(10)$ GUTs with hidden sector consisting of fermionic and bosonic $\mathrm{SO}(10)$ singlets and flavor symmetries. The framework allows to disentangle the CKM physics responsible for the CKM mixing and different mass hierarchies of quarks and leptons and the neutrino new physics which produces smallness of neutrino masses and large lepton mixing. The framework leads naturally to the relation $U_{\mathrm{PMNS}} \sim V_{\mathrm{CKM}}^{\dagger} U_{0}$, where structure of $U_{0}$ is determined by the flavor symmetry. The key feature of the framework is that apart from the Dirac mass matrices $m_{D}$, the portal mass matrix $M_{D}$ and the mass matrix of singlets $M_{S}$ are also involved in generation of the lepton mixing. This opens up new possibilities to realize the flavor symmetries and explain the data. Using $A_{4} \times Z_{4}$ as the flavor group, we systematically explore the flavor structures which can be obtained in this framework depending on field content and symmetry assignments. We formulate additional conditions which lead to $U_{0} \sim U_{\mathrm{TBM}}$ or $U_{\mathrm{BM}}$. They include (i) equality (in general, proportionality) of the singlet flavons couplings, (ii) equality of their VEVs; (iii) correlation between VEVs of singlets and triplet, (iv) certain VEV alignment of flavon triplet(s). These features can follow from additional symmetries or be remnants of further unification. Phenomenologically viable schemes with minimal flavon content and minimal number of couplings are constructed.

Keywords: GUT, Neutrino Physics, Beyond Standard Model, Quark Masses and SM Parameters

ARXIV EPRINT: 1604.03977 


\section{Contents}

1 Introduction $\quad 2$

2 Neutrino mass and mixing matrices 4

2.1 The framework 4

2.2 Yukawa couplings and mass matrices 4

2.3 Screening and partial screening $\quad 7$

2.4 Symmetry and flavor structures $\quad 8$

$\begin{array}{ll}2.5 & \text { Factorization of mixing matrix } \\ & 13\end{array}$

$\begin{array}{lll}2.6 & \text { Additional symmetries and VEV alignments } & 15\end{array}$

3 Schemes with $S$ transforming as a triplet of $A_{4} \quad 16$

$\begin{array}{ll}3.1 \text { Singlet-singlet flavon scheme } & 17\end{array}$

3.2 Triplet-triplet flavon scheme 20

$\begin{array}{ll}3.3 \text { Singlet-triplet flavon scheme } & 21\end{array}$

3.4 Triplet-singlet flavon scheme 23

3.5 Scheme with mixed contributions: the TBM mixing 23

3.6 Scheme with mixed contributions: the BM mixing 26

4 Schemes with $S$ transforming as singlets of $A_{4} \quad 28$

4.1 Scheme with $h_{10}=0 \quad 28$

4.2 Scheme with $\vec{y}_{10}=0 \quad 32$

5 Generalizations $\quad 33$

5.1 Effects of linear seesaw 33

5.2 Extended hidden sector 34

$\begin{array}{lll}5.3 & \text { On the CKM new physics } & 35\end{array}$

$\begin{array}{lll}6 & \text { Conclusion } & 37\end{array}$

$\begin{array}{lll}\text { A } \boldsymbol{A}_{4} \text { group } & 40\end{array}$

$\begin{array}{ll}\text { B Eigenvalues of } M_{33}^{\varphi} & 40\end{array}$

$\begin{array}{ll}\text { C Diagonalization of } M_{33}^{\xi} & 41\end{array}$

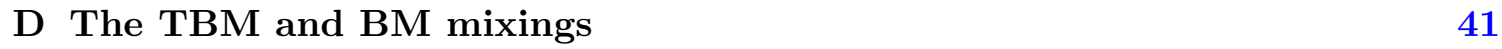




\section{Introduction}

There are several hints in favor of a framework with Grand unification, hidden sector at the string/Planck scale, and flavor symmetries acting both in visible and hidden sectors:

1. Grand Unification Theories (GUT) are still one of the highly motivated and very appealing scenarios of physics beyond the Standard Model (SM) [1,2]. In particular, the GUT models based on $\mathrm{SO}(10)[3,4]$ beside the gauge coupling unification and charge quantization accommodate exactly the quantum numbers of all known SM fermions, plus right-handed $(\mathrm{RH})$ neutrinos. This can not be accidental. The $\mathrm{SO}(10)$ GUT also provides natural realizations of the high scale seesaw mechanism [5-9], and therefore explanation of the smallness of neutrino masses.

2. In fact, the required mass scale of the $\mathrm{RH}$ neutrinos from the seesaw mechanism, $M_{N} \sim m_{D}^{2} / m_{\nu} \sim\left(10^{8}-10^{14}\right) \mathrm{GeV}$, where $m_{D}$ is a typical Dirac mass of the quarks and charged leptons, is somehow below the GUT scale. Nevertheless, the scale of $M_{N}$ can be made of the GUT scale $M_{\mathrm{GUT}}$ via the seesaw mechanism, if additional fermion singlets $S$ exist with masses at the Planck scale $M_{\mathrm{Pl}}: M_{N} \sim M_{\mathrm{GUT}}^{2} / M_{\mathrm{Pl}}$. Consequently, the double seesaw mechanism is realized for light neutrino masses [10].

3. Generically, the unification is at odd with strong difference of mixings and masses of quarks and leptons. Partially, the difference of mixings can be related to the smallness of neutrino mass [11-13] and the latter can originate again from the seesaw mechanism.

4. The approximate tri-bimaximal (TBM) lepton mixing [14] can be considered as an indication of existence of the flavor symmetry behind [15-17]. Furthermore, simple discrete groups, like $A_{4}[18,19]$, have certain properties which correspond to features of the TBM mixing [20-25]. In $\mathrm{SO}(10)$, realization of such a symmetry faces serious problems in view of strongly different mixing pattern in the quark sector [26, 27]. For some realizations with $A_{4}$ in $\mathrm{SO}(10)$ GUT, see e.g. [28-30].

5. Although being strongly different, the quark and lepton mixings can be related. Indeed, experimental data confirm the following connection between the quark, $V_{\mathrm{CKM}}$, and lepton, $U_{\mathrm{PMNS}}$, mixing matrices [31-42]

$$
U_{\mathrm{PMNS}} \simeq V_{\mathrm{CKM}}^{\dagger} U_{0} .
$$

Here $U_{0}$ is the matrix of special form, like the TBM mixing matrix $U_{\text {TBM }}$ or the bi-maximal (BM) mixing $U_{\mathrm{BM}}$ [43-47], dictated by symmetry and related to some new structures of theory which affect the lepton sector only. Eq. (1.1) gives a relation between the 1-3 mixing, 2-3 mixing and CKM mixing, which is in a good agreement with data [48]. In the lowest-order (only Cabibbo angle $\theta_{C}$ in $V_{\mathrm{CKM}}$, and maximal $2-3$ mixing) the relation reduces to $\sin ^{2} \theta_{13}=0.5 \sin ^{2} \theta_{C}[32,49]$.

The relation (1.1) implies Grand unification, which transfers information about mixing from the quark to lepton sector. 
6. The double seesaw mechanism allows to explain difference of mixing patterns in the quark and lepton sectors and reproduce the relation (1.1). It reveals new possibilities to realize flavor symmetries. Striking differences in the quark and lepton sectors follow essentially from the mixing of new fermion singlets $S$ with neutrino (left- and righthanded) components of 16-plet, thus providing the main source of the lepton mixing.

Origin of singlets $S$ can be related to further extension of the gauge symmetry: $\mathrm{SO}(10) \rightarrow E_{6}$, in which new singlets are additional neutral components of 27-plets [50]. Alternatively, singlet fermions $S$ can be components of the hidden sector composed of the fermionic and bosonic singlets of $\mathrm{SO}(10)$. Hidden sector may have its own gauge symmetries. Sterile neutrinos, if exist, particles of dark matter, axions, etc., can also originate from this sector.

It seems that the existing data on masses and mixing are too complicated to be explained all at once, from a few principles and a few parameters. So, according to eq. (1.1), probably two different (but somehow connected) types of new physics are involved: the "CKM new physics" responsible for the CKM mixing and difference of hierarchies of Dirac masses, and the "neutrino new physics", which explains smallness of neutrino masses and large mixing with its form dictated by symmetry. The neutrino new physics is related to the hidden sector and properties of the portal interactions which connect the visible sector and the hidden one [51-53].

This framework has been explored to some extent previously. That includes (i) the seesaw enhancement of lepton mixing due to coupling with new singlets [11], (ii) screening of the Dirac structures, which means that the mixing of light neutrinos is essentially related to mixing in the singlet sector $S: m_{\nu} \propto M_{S}$ [11,54], (iii) stability of this screening with respect to renormalization group (RG) running [54], renormalization of the relation (1.1) [55], (iv) symmetry origins (groups $T_{7}$ and $\Sigma_{81}$ have been used) of the screening, as well as partial screening [56], (v) lepton mixing from the symmetries of the hidden sector [48].

In this paper we further elaborate on the framework with emphasis on new mechanisms of getting flavor structures from symmetries. We systematically explore the fermion masses and mixing matrices which can be obtained in the framework. Interplay of the flavor and GUT symmetries is crucial. We mainly discuss here the symmetry aspects and formulate conditions of the vacuum alignment which eventually should be realized in complete model buildings.

The paper is organized as follows. In section 2 we study properties of the mass matrices involved in generation of the neutrino masses and mixing in this framework. We elaborate on the main elements and building blocks of the schemes of lepton mixing. In section 3 we present the simplest viable schemes with fermion singlets $S$ transforming under three-dimensional representation 3 of $A_{4}$. In section 4 the schemes are considered with $S$ transforming under one-dimensional representations $\mathbf{1}, \mathbf{1}^{\prime}, \mathbf{1}^{\prime \prime}$ of $A_{4}$. Section 5 is devoted to various generalizations: effect of linear seesaw, contribution from additional singlets, elements of the CKM physics. We summarize our results in section 6. Some technical details are presented in the appendix. 


\section{Neutrino mass and mixing matrices}

\subsection{The framework}

Let us first summarize main ingredients of the framework outlined in the introduction. We consider the $\mathrm{SO}(10)$ gauge model with three generations of fermions and the following additional features:

- All the chiral fermions (including the right-handed neutrino) of each generation are accommodated in a 16-dimensional spinor representation, $16_{F i}(i=1,2,3)$.

- Three or more singlet fermions $S_{i}$ exist. They mix with neutrinos due to Yukawa interactions with 16-plets of Higgs scalars $\overline{16}_{H}$.

- Only low-dimension representations of Higgs fields, $10_{H}, \overline{16}_{H}$ and $1_{H}$, give masses to fermions. High dimensional representations $126_{H}$ and $120_{H}$ are absent.

- Approximate flavor symmetry exists with fermions $16_{F i}, S_{j}$ carrying non-zero flavor charges. For definiteness we consider the simplest discrete group which has irreducible three-dimensional representations, $A_{4}$. Various features obtained from this symmetry can be also reproduced with other groups.

- Three generations of the matter fields, $16_{F i}$, transform as triplet of $A_{4}$ in most of the cases. We explore different symmetry assignments for three singlets $S_{i},\left(\mathbf{3}, \mathbf{1}, \mathbf{1}^{\prime}, \mathbf{1}^{\prime \prime}\right)$.

- A number of flavons (scalar fields, $\mathrm{SO}(10)$ singlets) exist, which have non-trivial transformations with respect to $A_{4}$ and acquire vacuum expectation values (VEV), thus breaking the symmetry.

- The Higgs multiplets, $10_{H}$ and $16_{H}$, are singlets of $A_{4}$, and therefore factor out of the flavor structure in the simplest cases. This reduces the complexity in obtaining certain form of mass matrices and mixing from flavor symmetries. Still, $10_{H}$ and $16_{H}$ may carry charges of additional symmetries, in which case they will affect the flavor.

We do not specify here the $\mathrm{SO}(10)$ symmetry breaking, although the corresponding Higgs multiplets can also take part in generation of the fermion masses. Rather than constructing complete models, we will explore systematically the flavor structures which can be obtained in the formulated framework.

\subsection{Yukawa couplings and mass matrices}

The Lagrangian has three types of Yukawa couplings,

$$
\mathcal{L}=\mathcal{L}_{D}+\mathcal{L}_{N S}+\mathcal{L}_{S},
$$

which produce masses of fermions: 
1. The visible (Dirac) sector Yukawa interactions

$$
\mathcal{L}_{D}=Y_{10}^{i j a} \cdot 16_{F i} 16_{F j} 10_{H}^{a}
$$

generate after symmetry breaking the usual Dirac masses of fermions. With just one $10_{H}$ these couplings produce Dirac mass matrices of the up-type quark, down-type quark, charge lepton mass matrices and neutrinos of the same form:

$$
m_{u}=m_{D}=Y_{10} v_{u}, \quad m_{d}=m_{l}=Y_{10} v_{d}
$$

where $v_{u} v_{d}$ are the VEVs of $10_{H}$. No quark mixing is generated at this level. Nevertheless, such a mixing can be produced if two (or more) different $10_{H}^{a}(a=u, d)$ are introduced (see section 5.3).

2. The portal interactions,

$$
\mathcal{L}_{N S}=Y_{16}^{i j} \cdot 16_{F i} S_{j} \overline{16}_{H},
$$

generate mass matrices of Dirac type for the $\mathrm{LH}$ and $\mathrm{RH}$ neutrinos from $16_{F i}$ and singlets $S_{j}$

$$
m^{\prime}=Y_{16} v_{L}, \quad M_{D}=Y_{16} v_{R} .
$$

Here $v_{L}, v_{R}$ are the VEVs of the $\mathrm{SU}(2)_{L}$ and $\mathrm{SU}(2)_{R}$ doublets from $\overline{16}_{H}$. As long as only one Higgs 16-plet generates $M_{D}$ and $m^{\prime}$, the two mass matrices are proportional to each other:

$$
m^{\prime}=\frac{v_{L}}{v_{R}} M_{D} .
$$

3. The hidden sector interactions,

$$
\mathcal{L}_{S}=Y_{1}^{i j} \cdot S_{i} S_{j} 1_{H}+M_{S}^{0 i j} S_{i} S_{j},
$$

produce the mass matrix of singlets

$$
M_{S}=Y_{1}\left\langle 1_{H}\right\rangle+M_{S}^{0} .
$$

Here $1_{H}$ fields are singlets under both flavon symmetry and $\mathrm{SO}(10)$, which may not be necessary and are assumed to be absorbed into flavons from now on. The masses of singlets can be generated at the renormalizable level, and also bare mass terms $M_{S}^{0}$ can appear for some or all singlets if it is not forbidden by flavor symmetry.

Notice that here the Yukawa couplings are effective couplings - functions of VEVs of flavon fields, $\Phi$ :

$$
Y_{k}^{i j a}=Y_{k}^{i j a}(\langle\Phi\rangle) .
$$

If $\langle\Phi\rangle \ll \Lambda_{f}$, where $\Lambda_{f}$ is a scale of flavor physics, the constants can be expanded as

$$
Y_{k}^{i j a}=y_{k}^{i j a}+\left[y_{k} \frac{\langle\Phi\rangle}{\Lambda_{f}}\right]^{i j a}+\left[y_{k}^{2} \frac{\langle\Phi\rangle^{2}}{\Lambda_{f}^{2}}\right]^{i j a}+\ldots, \quad k=10,16,1 .
$$

In what follows we will take the lowest-order approximation in $\langle\Phi\rangle / \Lambda_{f}$. 
The mass matrix of neutral leptons generated by interactions (2.2), (2.4) and (2.6) in the basis $(\nu, N, S)$, where $N \equiv \nu^{c}$, equals

$$
\left(\begin{array}{ccc}
0 & m_{D} & m^{\prime} \\
m_{D}^{T} & 0 & M_{D} \\
m^{\prime T} & M_{D}^{T} & M_{S}
\end{array}\right) .
$$

Given the Yukawa interactions introduced above, the matrices $m_{D}$ and $M_{S}$ are symmetric: $m_{D}=m_{D}^{T}$ and $M_{S}=M_{S}^{T}$, while $m^{\prime}$ and $M_{D}$ may have a non-symmetric form. The mass terms are defined as $\nu^{T} m_{D} C^{-1} N, N^{T} M_{D} C^{-1} S$, etc., and $C \equiv i \gamma^{0} \gamma^{2}$. For

$$
m_{D}, m^{\prime} \ll M_{D}, \quad M_{S}
$$

the diagonalization of (2.7) leads to the light neutrino mass matrix

$$
m_{\nu} \simeq m_{D} \frac{1}{M_{D}^{T}} M_{S} \frac{1}{M_{D}} m_{D}^{T}-\left(m_{D} \frac{1}{M_{D}^{T}} m^{T}+m^{\prime} \frac{1}{M_{D}} m_{D}^{T}\right),
$$

where the first term is the double seesaw contribution, and two others correspond to the linear seesaw contribution. In the case of a single $\overline{16}_{H}$, according to eq. (2.5) the mass matrix (2.8) becomes

$$
m_{\nu} \simeq m_{D} \frac{1}{M_{D}^{T}} M_{S} \frac{1}{M_{D}} m_{D}^{T}-\frac{2 v_{L}}{v_{R}} m_{D}
$$

Given that $m_{\nu} \sim \mathrm{O}(0.1) \mathrm{eV}$ and $v_{L}<246 \mathrm{GeV}$, it is possible to define a critical mass scale

$$
M_{\mathrm{cr}}=4 \times 10^{14}\left(\frac{v_{L} \cdot Y_{16}}{246 \mathrm{GeV}}\right)^{2} \mathrm{GeV},
$$

so that for $M_{S} \ll M_{\mathrm{cr}}$ the "linear-seesaw" dominates, whereas for $M_{S} \gg M_{\mathrm{cr}}$, the "doubleseesaw" gives the main contribution. In our framework with $M_{S} \sim M_{\mathrm{Pl}}$ the "doubleseesaw" dominates and the "linear-seesaw" term may lead to small corrections.

According to eq. (2.3) and $m_{D} \propto m_{l}$, no mixing is produced by the Dirac mass matrices. In contrast, due to extended structure of the mass matrix of neutral leptons eq. (2.7), $m_{\nu}$ differs from $m_{l}$, and so the lepton mixing is generated. In the basis where the Dirac mass matrices are diagonal, $m_{D}=m_{D}^{\text {diag }}$, the origin of lepton mixing is the structure

$$
m_{D}^{\text {diag }} M_{D}^{-1 T} M_{S} M_{D}^{-1} m_{D}^{\text {diag }}
$$

In the lowest order, the Majorana mass term of right-handed neutrinos, $N^{T} C^{-1} N$, is absent, since we do not introduce 126 Higgs multiplet. Such a term can be generated by higher-dimension operator $(1 / \Lambda) 16_{F i} 16_{F j}\left(16_{H} 16_{H}\right)^{i j}$, or effectively generated by the decoupling of heavy particles with mass $\sim \Lambda$. It then gives $M_{N}=v_{R}^{2} / \Lambda$.

In our framework, the mass term $N^{T} C^{-1} N$ appears after integrating out $S$, which gives

$$
M_{N}=M_{D}^{T} M_{S}^{-1} M_{D}=v_{R}^{2} Y_{16}^{T} M_{S}{ }^{-1} Y_{16} .
$$

We assume that this contribution to $M_{N}$ dominates. Other contributions either are forbidden by certain auxiliary symmetry or have the same flavor structure as eq. (2.12).

Notice that since in our framework there is $m_{D} \ll M_{D}$, the RG running of Yukawa couplings may modify the expressions for neutrino masses non-negligibly, and thus may need to be taken into account [57-61]. 


\subsection{Screening and partial screening}

The double seesaw allows to realize the screening of the Dirac structures [11, 54], which will be used in many examples presented in this paper. If

$$
m_{D} \propto M_{D}^{T}
$$

then according to eq. (2.9)

$$
m_{\nu} \propto M_{S}
$$

i.e. structure of the light neutrino mass matrix is given by that of $M_{S}$, thus disentangling properties of neutrinos and quarks completely. The form of $M_{S}$ can strongly differ from those of $m_{D}$ and $M_{D}$, thus leading to very different lepton mixing.

Suppose that the screening condition is fulfilled in the basis where $m_{D} \propto M_{D}^{T}$ are diagonal due to some certain symmetry, i.e. in the flavor basis, and that in this basis the singlet mass matrix is $M_{S}$. Then according to (2.14) the mixing is determined exclusively by $M_{S}$.

If the screening condition (2.13) is fulfilled in some other basis (again determined by symmetry) where $m_{l}=m_{D}$ is non-diagonal and the singlet matrix is $M_{S}^{\prime}$, then $m_{\nu} \propto M_{S}^{\prime}$. But now the lepton mixing gets contributions both from $m_{D}$ and $M_{S}^{\prime}$. We present schemes which realize both possibilities.

Let us consider the screening condition in more details. We define the unitary transformations $U_{l}, V_{R}, V_{S}$ and $V_{N}$ which diagonalize the mass matrices $m_{D}$ and $M_{D}$, so that

$$
m_{D}=U_{l} m_{D}^{\text {diag }} V_{R}^{\dagger}, \quad M_{D}^{T}=V_{S} M_{D}^{\text {diag }} V_{N}^{\dagger}
$$

Then the screening matrix (factor) equals

$$
m_{D}\left(M_{D}^{T}\right)^{-1}=U_{l} m_{D}^{\text {diag }} V_{R}^{\dagger} V_{N}\left(M_{D}^{\text {diag }}\right)^{-1} V_{S}^{\dagger} .
$$

The screening (2.13) implies that

$$
V_{R}=V_{N}, \quad m_{D}^{\text {diag }} \propto M_{D}^{\text {diag }}, \quad U_{l}=V_{S} .
$$

If only the first condition (the same rotations of the $\mathrm{RH}$ neutrino components) and the second one are fulfilled we obtain

$$
m_{D}\left(M_{D}^{T}\right)^{-1}=U_{l} V_{S}^{\dagger} \neq I .
$$

This partial screening gives additional contribution to the neutrino mixing. Due to symmetry the matrices $U_{l}$ and $V_{S}$ can be related in such a way that eq. (2.15) produces a matrix of special form, which then leads to the required form of total mixing matrix (see section 3.5). The screening can be due to further embedding of singlets $S$ in extended gauge symmetry or due to common flavor symmetries.

Some additional physics (which we call the CKM physics) can lead to the misalignment of mass matrices for up- and down- type quarks. Due to GUT similar misalignment is expected between the neutrino Dirac matrix $m_{D}$ and the charged lepton matrix $m_{l} \neq m_{D}$. 
Then in the presence of screening $m_{D} \propto M_{D}^{T}=$ diag the PMNS mixing gets the CKM type contribution $\sim V_{\mathrm{CKM}}$ from $m_{l}$ and another one, $U_{S}$, from $M_{S}$ :

$$
U_{\mathrm{PMNS}}=V_{\mathrm{CKM}}^{\dagger} U_{S} .
$$

This allows to maximally disentangle the CKM and neutrino new physics and realize the scenario in eq. (1.1).

\subsection{Symmetry and flavor structures}

The flavor symmetry imposes restrictions on the Yukawa couplings $Y_{k}^{i j}$, and thus forbids some of them or leads to relations between them. For definiteness we use $A_{4}$ flavor group (see appendix A for details). Various structures we obtain in this paper can be reproduced in models with other symmetry groups which include representations $\mathbf{3}, \mathbf{1}, \mathbf{1}^{\prime}, \mathbf{1}^{\prime \prime}$. On the other hand, with additional symmetries it is possible to produce in our framework features from other symmetry groups.

We introduce flavon triplets $\xi$ and singlets $\vec{\varphi}=\left(\varphi, \varphi^{\prime}, \varphi^{\prime \prime}\right)$, which acquire the VEV $\langle\xi\rangle \equiv\left(u_{1}, u_{2}, u_{3}\right)$ and $\left(\langle\varphi\rangle,\left\langle\varphi^{\prime}\right\rangle,\left\langle\varphi^{\prime \prime}\right\rangle\right) \equiv\left(v, v^{\prime}, v^{\prime \prime}\right)$. The general form of a fermion mass term is $F_{i} F_{j}$, where the fermionic multiplets $F_{i}=16_{F}, S$. Depending on symmetry assignment of $F_{i}$ and for general sets of the flavon fields, which include the flavon triplets $\xi$ and singlets $\vec{\varphi}$, we obtain three types of mass matrices:

1. $F_{1} \sim \mathbf{3}, F_{2} \sim \mathbf{3}$. The most general coupling with flavons invariant under $A_{4}$ is

$$
m_{0}\left(F_{1} F_{2}\right)+\vec{y}\left(F_{1} F_{2}\right)_{\varphi} \vec{\varphi}+h\left(F_{1} F_{2}\right) \xi
$$

where we use notations

$$
\vec{y}\left(F_{1} F_{2}\right)_{\varphi} \vec{\varphi} \equiv y\left(F_{1} F_{2}\right) \varphi+y^{\prime}\left(F_{1} F_{2}\right)^{\prime \prime} \varphi^{\prime}+y^{\prime \prime}\left(F_{1} F_{2}\right)^{\prime} \varphi^{\prime \prime} .
$$

Here $\vec{y} \equiv\left(y, y^{\prime}, y^{\prime \prime}\right)$ and $(Q)_{\varphi}\left(Q=F_{1} F_{2}\right.$ in this case $)$ is the field operator which produces one-dimensional representations of $A_{4}$ together with a given $\varphi$.

The mass term $m_{0}\left(F_{1} F_{2}\right)$ without flavons can be forbidden by symmetries. The interactions (2.16) lead to the mass terms $F_{1} M_{33} F_{2}^{T}+$ h.c. with

$$
M_{33}=M_{33}^{\text {diag }}+M_{33}^{\text {off }}=m_{0} \mathbb{1}+M_{33}^{\varphi}+M_{33}^{\xi},
$$

where the off-diagonal part is generated by flavon triplet, whereas the diagonal one

$$
M_{33}^{\text {diag }}=m_{0} \mathbb{1}+M_{33}^{\varphi}
$$

is due to coupling with the flavon singlets of $A_{4}$ and flavonless contribution. Three terms in (2.18) are generated by different flavons. Depending on additional symmetry assignments one, or two, or all three terms can contribute. Explicitly,

$$
M_{33}^{\varphi}=\left(\begin{array}{ccc}
y v+y^{\prime} v^{\prime}+y^{\prime \prime} v^{\prime \prime} & 0 & 0 \\
0 & y v+\omega y^{\prime} v^{\prime}+\omega^{2} y^{\prime \prime} v^{\prime \prime} & 0 \\
0 & 0 & y v+\omega^{2} y^{\prime} v^{\prime}+\omega y^{\prime \prime} v^{\prime \prime}
\end{array}\right)
$$


Notice that for all equal values $\left(y v=y^{\prime} v^{\prime}=y^{\prime \prime} v^{\prime \prime}\right), M_{33}^{\operatorname{diag}}=\operatorname{diag}\left(m_{0}+3 y v, m_{0}, m_{0}\right)$, which can be a good first approximation in some cases. For real parameters $\mu \equiv$ $m_{0}+y v, \mu^{\prime} \equiv y^{\prime} v^{\prime}$ and $\mu^{\prime \prime} \equiv y^{\prime \prime} v^{\prime \prime}$ two eigenstates are degenerate:

$$
\left|M_{2}\right|=\left|M_{3}\right|=\left[\left(\mu+\mu^{\prime}+\mu^{\prime \prime}\right)^{2}-3\left(\mu \mu^{\prime}+\mu^{\prime} \mu^{\prime \prime}+\mu \mu^{\prime \prime}\right)\right]^{1 / 2} .
$$

This important feature together with certain forms of the off-diagonal part can lead to maximal mixing. General situation is analyzed in the appendix B.

In the absence of the bare mass $m_{0}$, the eigenvalues of matrix (2.19) can be written as

$$
\left(M_{1}, M_{2}, M_{3}\right)^{T}=\sqrt{3} U_{\omega}\left(y v, y^{\prime} v^{\prime}, y^{\prime \prime} v^{\prime \prime}\right)^{T}
$$

where

$$
U_{\omega}=\frac{1}{\sqrt{3}}\left(\begin{array}{ccc}
1 & 1 & 1 \\
1 & \omega & \omega^{2} \\
1 & \omega^{2} & \omega
\end{array}\right)
$$

is the magic matrix. The matrix $U_{\omega}$ is unitary, $U_{\omega} U_{\omega}^{\dagger}=I$ and the inverse $U_{\omega}^{-1}=$ $U_{\omega}^{\dagger}=U_{\omega}^{*}$, can be obtained by substitution $\omega \leftrightarrow \omega^{2}$.

Then the condition

$$
y v \approx \omega^{2} y^{\prime} v^{\prime} \approx \omega y^{\prime \prime} v^{\prime \prime}
$$

leads to the hierarchy of masses:

$$
m_{3}=3 y v \gg m_{2}, m_{1} .
$$

The hierarchy (mass splitting) between the lighter states, $m_{2} \gg m_{1}$, can be obtained by special tuning of relations in eq. (2.22) or introduction of other contributions, e.g. from the linear seesaw terms.

In general, it is difficult to get correlation (2.22) between VEVs and Yukawa couplings. The simplest realization would be two separate equalities:

$$
y \approx y^{\prime} \approx y^{\prime \prime}
$$

as a result of additional symmetry, or the fact that singlets originate from breaking of representation $\mathbf{3}$ and

$$
v \approx \omega^{2} v^{\prime} \approx \omega v^{\prime \prime}
$$

as a consequence of special forms of the potential.

In eq. (2.18) the off-diagonal matrix equals

$$
M_{33}^{\xi}=h\left(\begin{array}{ccc}
0 & u_{3} & u_{2} \\
\ldots & 0 & u_{1} \\
\ldots & \ldots & 0
\end{array}\right)
$$

It has the form of the Zee-Wolfenstein mass matrix $[62,63]$ with the eigenvalues satisfying the equality $m_{1}+m_{2}+m_{3}=0$. For arbitrary values of $u_{i}$, the diagonalization 
matrix and eigenvalues of $M_{33}^{\xi}$ are presented in appendix C, and here we consider some special cases.

If $u_{1}=u_{2}$, the matrix can be diagonalized by maximal 2-3 rotation and vanishing $\theta_{13}$. Then for the $1-2$ mixing we obtain $\sin ^{2} 2 \theta_{12}=8 u_{1}^{2} /\left(8 u_{1}^{2}+u_{2}^{2}\right)$. If all VEVs are equal, $u_{1}=u_{2}=u_{3}$, then $\sin ^{2} 2 \theta_{12}=8 / 9$, which corresponds to the TBM mixing.

Let us consider a special case of the total matrix $M_{33}$ (2.18), which will appear often in our constructions. If $u_{1}=u_{2}=u_{3} \equiv u$ and also the diagonal elements are all equal, we have

$$
M_{33}^{\text {special }}=\left(\begin{array}{ccc}
\mu & \beta & \beta \\
\ldots & \mu & \beta \\
\ldots & \ldots & \mu
\end{array}\right)
$$

where $\beta \equiv h u$ [64]. Such a matrix can be obtained when $\varphi^{\prime}$ and $\varphi^{\prime \prime}$ do not contribute to masses, or when the bare mass terms or flavonless operators dominate. This matrix having the TBM form is diagonalized by $U_{\mathrm{TBM}}$, see appendix $\mathrm{D}$. The eigenvalues equal

$$
\lambda_{1}=\lambda_{3}=\mu-\beta, \quad \lambda_{2}=\mu+2 \beta,
$$

of which two are equal and the sum is given by

$$
\sum_{i} \lambda_{i}=3 \mu
$$

For $\mu=0$ we have $\sum_{i} \lambda_{i}=0$, and therefore $M_{33}^{\text {special }}(\mu=0)$ can not be used to describe masses of quarks or charged leptons.

Degeneracy of the two eigenstates implies that the diagonalization matrix is not unique, and actually there is infinite ambiguity in diagonalization related to rotation in the space of eigenstates with equal masses. In particular, the matrix (2.24) can be unitarily diagonalized by the magic matrix as $^{1}$

$$
U_{\omega}^{*} M_{33}^{\text {special }} U_{\omega}
$$

The diagonalization gives $\lambda_{1}=\mu+2 \beta$ and $\lambda_{2}=\lambda_{3}=\mu-\beta$, which coincides with eq. (2.25) up to permutation.

The ambiguity is removed when the degeneracy of eigenvalues is broken. Let us consider correction matrices of the form

$$
\Delta M=M_{0}\left(\begin{array}{lll}
1 & 0 & 0 \\
0 & 0 & 1 \\
0 & 1 & 0
\end{array}\right) \quad \text { or } \quad \Delta M^{\prime}=M_{0}^{\prime}\left(\begin{array}{lll}
0 & 0 & 1 \\
1 & 0 & 0 \\
0 & 1 & 0
\end{array}\right),
$$

\footnotetext{
${ }^{1}$ Note that in terms of the SM components, the Dirac mass terms read

$$
\left(l_{L}^{c}\right)^{T} C m_{D} l_{L}+l_{L}^{T} m_{D} C l_{L}^{c}+\text { h.c., }
$$

where $l_{L}$ and $\left(l^{c}\right)_{L}$ are the left and the right components, and $m_{D}$ is a symmetric matrix. Then the unitary diagonalization

$$
U_{\omega}^{*} m_{D} U_{\omega}=\operatorname{diag}\left(m_{1}, m_{2}, m_{3}\right),
$$

would imply that $l_{L}$ and $\left(l^{c}\right)_{L}$ transform differently, but $l_{L}$ and $l_{R}$ transform in the same way.
} 
of which both can arise from a $Z_{3}$ symmetry, with different charge assignments. The former symmetric matrix fixes the diagonalization matrix to $U_{\mathrm{TBM}}$, whereas the latter one fixes it to $U_{\omega}$. Note that $U_{\omega}$ can only be obtained from non-symmetric corrections, such as $\Delta M^{\prime}$.

It is easy to get maximal mixing from $M_{33}$, which will be often needed for our constructions below. For instance, the maximal 2-3 mixing can be obtained if $\langle\xi\rangle=$ $\left(u_{1}, 0,0\right)$ and $y^{\prime} v^{\prime}=y^{\prime \prime} v^{\prime \prime}$, or $\vec{y}=0$. For maximal 1-3 mixing one needs $\langle\xi\rangle=\left(0, u_{2}, 0\right)$ and $y^{\prime} v^{\prime}=\omega y^{\prime \prime} v^{\prime \prime}$.

In general, by varying $y v, y^{\prime} v^{\prime}, y^{\prime \prime} v^{\prime \prime}$ as well as $h u_{i}$, one can obtain an arbitrary symmetric matrix for $M_{33}$.

2. $F_{1} \sim \mathbf{3}$ and $\vec{F}_{2} \equiv\left(F_{2}, F_{2}^{\prime}, F_{2}^{\prime \prime}\right) \sim\left(\mathbf{1}, \mathbf{1}^{\prime}, \mathbf{1}^{\prime \prime}\right)$. The most general couplings with flavons

$$
\vec{h}\left(F_{1} \xi\right)_{F_{2}} \vec{F}_{2} \equiv h\left(F_{1} \xi\right) F_{2}+h^{\prime}\left(F_{1} \xi\right)^{\prime \prime} F_{2}^{\prime}+h^{\prime \prime}\left(F_{1} \xi\right)^{\prime} F_{2}^{\prime \prime}
$$

generate the mass term $F_{1} M_{31} F_{2}^{T}$ with

$$
M_{31}=\left(\begin{array}{ccc}
h u_{1} & h^{\prime} u_{1} & h^{\prime \prime} u_{1} \\
h u_{2} & h^{\prime} u_{2} \omega & h^{\prime \prime} u_{2} \omega^{2} \\
h u_{3} & h^{\prime} u_{3} \omega^{2} & h^{\prime \prime} u_{3} \omega
\end{array}\right) .
$$

Obviously, only flavon triplets contribute to $M_{31}[65,66]$. This matrix can be represented in the form

$$
M_{31}=\sqrt{3} D_{u} U_{\omega} D_{h},
$$

where $D_{u} \equiv \operatorname{diag}\left(u_{1}, u_{2}, u_{3}\right)$ and $D_{h} \equiv \operatorname{diag}\left(h, h^{\prime}, h^{\prime \prime}\right)$.

Additional symmetries related to transformations of components of $\vec{F}_{2}$ may lead, e.g. to $h=h^{\prime}=h^{\prime \prime}$, thus further reducing the number of free parameters in $D_{h}$. If two or all three fermions in $\vec{F}_{2}$ have the same symmetry assignments, including charges under $A_{4}$, e.g. $\vec{F}_{2} \sim\left(\mathbf{1}, \mathbf{1}^{\prime}, \mathbf{1}^{\prime}\right)$, we would obtain a singular mass matrix $M_{31}$ with two or three columns proportional to each other.

In general, the matrix (2.28) is non-symmetric. It can be made symmetric if $D_{h} \propto D_{u}$ (or equivalently, $u_{i} \propto h^{i}$ ), which can be obtained when $h^{i}$ themselves are given by VEVs of some new fields.

3. $\vec{F}_{1} \equiv\left(F_{1}, F_{1}^{\prime}, F_{1}^{\prime \prime}\right) \sim\left(\mathbf{1}, \mathbf{1}^{\prime}, \mathbf{1}^{\prime \prime}\right), \vec{F}_{2} \sim\left(\mathbf{1}, \mathbf{1}^{\prime}, \mathbf{1}^{\prime \prime}\right)$. For the same set of the fermionic singlets, i.e. $\vec{F}_{2}=\vec{F}_{1}$, the most general coupling with flavons $\varphi, \varphi^{\prime}, \varphi^{\prime \prime}$ is given by

$$
\begin{aligned}
& m_{0} F_{1} F_{1}+2 \tilde{m}_{0} F_{1}^{\prime} F_{1}^{\prime \prime}+\left(y_{11} F_{1} F_{1}+2 y_{23} F_{1}^{\prime} F_{1}^{\prime \prime}\right) \varphi+\left(y_{22} F_{1}^{\prime} F_{1}^{\prime}+2 y_{13} F_{1} F_{1}^{\prime \prime}\right) \varphi^{\prime} \\
& \quad+\left(2 y_{12} F_{1} F_{1}^{\prime}+y_{33} F_{1}^{\prime \prime} F_{1}^{\prime \prime}\right) \varphi^{\prime \prime}+\text { h.c. }
\end{aligned}
$$

It leads to the mass terms $\vec{F}_{1} M_{11} \vec{F}_{1}^{T}$, where

$$
M_{11}=\left(\begin{array}{ccc}
m_{0}+y_{11} v & y_{12} v^{\prime \prime} & y_{13} v^{\prime} \\
\ldots & y_{22} v^{\prime} & \tilde{m}_{0}+y_{23} v \\
\ldots & \ldots & y_{33} v^{\prime \prime}
\end{array}\right) .
$$


For all free parameters, $M_{11}$ is an arbitrary symmetric matrix. One can get from eq. (2.31) maximal mixing or the TBM mixing immediately under the conditions

$$
y_{22} v^{\prime}=y_{33} v^{\prime \prime}, \quad y_{12} y_{22}=y_{13} y_{33}, \quad\left(m_{0}-\tilde{m}_{0}\right)+\left(y_{11}-y_{23}\right) v+\left(y_{12}-y_{33}\right) v^{\prime \prime}=0 .
$$

They are satisfied if, e.g. $m_{0}=\tilde{m}_{0}$, while all the couplings and all the VEVs are equal, respectively.

If the bare mass term dominates, it produces the structure with maximal 2-3 mixing which can be used to construct the TBM or BM mixing.

If all the couplings in eq. (2.31) are equal and the bare mass terms are absent being forbidden by additional symmetry, the matrix $M_{11}$ acquires a form

$$
M_{\omega}=\left(\begin{array}{lll}
a & c & b \\
c & b & a \\
b & a & c
\end{array}\right),
$$

where $a=h v, b=h v^{\prime}$ and $c=h v^{\prime \prime}$. The matrix $M_{\omega}$ is diagonalized by the magic matrix $U_{\omega}$ as $U_{\omega}^{T} M_{\omega} U_{\omega}$. The eigenvalues of $M_{\omega}$ in this case equal

$$
\left(m_{1}, m_{2}, m_{3}\right)^{T}=\sqrt{3} U_{\omega}^{*}(a, b, c)^{T},
$$

and there is no mass (eigenvalue) degeneracy provided that none of $\alpha, \omega \alpha$, and $\omega^{2} \alpha$ is real, where $\alpha \equiv a b^{*}+b c^{*}+c a^{*}$. Notice that eq. (2.33) is the only form of symmetric matrix which produces the mixing $U_{l}=U_{\omega}^{*}{ }^{2}$

Thus, $A_{4}$ with additional conditions (i.e. equalities of couplings and VEVs, which in turn imply some additional symmetries) allows to get $U_{\text {TBM }}$ or $U_{\omega}$. The latter appears from various interactions, and together with additional maximal 1-3 rotation, can again lead to $U_{\text {TBM. }}$. In this sense $A_{4}$ has features which are close to the TBM mixing.

For $16_{F} \sim \mathbf{3}$, the Dirac mass matrices are of $M_{33}$ form: $m_{D}=M_{33}^{(D)}$. With certain restrictions they can be reduced to $M_{33}^{\varphi}$ or $M_{33}^{\xi}$ type. If $S \sim \mathbf{3}$, all other matrices also have the form $M_{33}: M_{D}=M_{33}^{(P)}, M_{S}=M_{33}^{(S)}$ although values of elements of these matrices being functions of different couplings $(y, h)$ and VEVs $(v, u)$, are different. This is marked by different superscripts. Here also additional restrictions can select $M_{33}^{\text {diag }}$ or $M_{33}^{\xi}$. All these matrices are symmetric. The mass matrix of light neutrinos equals

$$
m_{\nu}=M_{33}^{(D)}\left[M_{33}^{(P) T}\right]^{-1} \cdot M_{33}^{(S)} \cdot\left[M_{33}^{(P)}\right]^{-1} M_{33}^{(D)} .
$$

For $S \sim\left(\mathbf{1}, \mathbf{1}^{\prime}, \mathbf{1}^{\prime \prime}\right)$ the portal and singlet mass matrices have different structures: $M_{D}=M_{31}^{(P)}$ and $M_{S}=M_{11}^{(S)}$. Correspondingly, the light neutrino mass matrix is given by

$$
m_{\nu}=M_{33}^{(D)}\left(M_{31}^{(P) T}\right)^{-1} M_{11}^{(S)}\left[M_{31}^{(P)}\right]^{-1} M_{33}^{(D)},
$$

\footnotetext{
${ }^{2}$ If $m_{D}$ is non-symmetric, which can happen in the presence of additional $120_{H}$ or $45_{H}$ Higgs multiplets, other forms are allowed to generate $U_{\omega}$.
} 


\begin{tabular}{|c|c|c|c|c|}
\hline $16_{F}$ under $A_{4}$ & $S$ under $A_{4}$ & Dirac $m_{D}$ & portal $M_{D}$ & singlet $M_{S}$ \\
\hline $\mathbf{3}$ & $\mathbf{3}$ & $M_{33}^{(D)}$ & $M_{33}^{(P)}$ & $M_{33}^{(S)}$ \\
\hline $\mathbf{3}$ & $\left(\mathbf{1}, \mathbf{1}^{\prime}, \mathbf{1}^{\prime \prime}\right)$ & $M_{33}^{(D)}$ & $M_{31}^{(P)}$ & $M_{11}^{(S)}$ \\
\hline$\left(\mathbf{1}, \mathbf{1}^{\prime}, \mathbf{1}^{\prime \prime}\right)$ & $\mathbf{3}$ & $M_{11}^{(D)}$ & $M_{13}^{(P)}$ & $M_{33}^{(S)}$ \\
\hline$\left(\mathbf{1}, \mathbf{1}^{\prime}, \mathbf{1}^{\prime \prime}\right)$ & $\left(\mathbf{1}, \mathbf{1}^{\prime}, \mathbf{1}^{\prime \prime}\right)$ & $M_{11}^{(D)}$ & $M_{11}^{(P)}$ & $M_{11}^{(S)}$ \\
\hline
\end{tabular}

Table 1. Combinations of mass matrices for various $A_{4}$ assignments.

and now $M_{D}$ is usually not symmetric. Using representation (2.29) we have

$$
m_{\nu}=\frac{1}{3} M_{33}^{(D)} D_{u}^{-1} \cdot U_{\omega}^{*} \cdot\left(D_{h}^{-1} M_{11}^{(S)} D_{h}^{-1}\right) \cdot U_{\omega}^{*} \cdot D_{u}^{-1} M_{33}^{(D)} .
$$

The screening can arise from $M_{33}^{(D)}\left(M_{33}^{(P) T}\right)^{-1}$ in eq. (2.34), or partial screening from $M_{33}^{(D)} D_{u}^{-1}$ in eq. (2.35), depending on the $A_{4}$ charges of fermion singlets.

Including also the possibility of $16_{F} \sim\left(\mathbf{1}, \mathbf{1}^{\prime}, \mathbf{1}^{\prime \prime}\right)$ we obtain four different combinations of mass matrices shown in the table 1 . In what follows we mostly focus on the first two possibilities with $16_{F} \sim \mathbf{3}$ and $S$ transforming as either $\mathbf{3}$ or $\left(\mathbf{1}, \mathbf{1}^{\prime}, \mathbf{1}^{\prime \prime}\right)$.

In general, any structure of $m_{\nu}$ can be obtained. So, additional restrictions are needed in order to produce specific flavor structures. Further structuring can be achieved if not all possible flavons are introduced when some couplings and VEVs of flavons are forbidden (zero) or are related to each other. The latter implies an additional symmetry, such as $Z_{2}$ or $Z_{4}$. If more than three fermionic singlets $S$ exist we can obtain more flavor structures assigning $S$ in triplet and singlet representations of $A_{4}: S \sim \mathbf{3}+\left(\mathbf{1}, \mathbf{1}^{\prime}, \mathbf{1}^{\prime \prime}\right)$. This extension will be considered in some details in section 5.2.

Finally, in the case of $16_{F}$ being singlets of $A_{4}, m_{D}=M_{11}^{(D)}$ in the form of eq. (2.31), and some new possibilities appear:

1. As we have marked already, equal Yukawa couplings (without bare masses) lead to the Dirac mass matrix of the form eq. (2.33). The later is diagonalized by $U_{\omega}$. Then maximal 1-3 mixing can follow from the portal and hidden sector interactions to produce the TBM mixing.

2. If $S$ are transforming as triplets of $A_{4}$, partial screening, i.e. $m_{D} / M_{D} \propto U_{\omega}$, can be obtained since in this case $M_{13}^{(P)} \sim M_{31}^{T} \sim D_{h} U_{\omega} D_{u}$. However, such a construction usually corresponds to a diagonal $m_{D}$, which is difficult to generate from $m_{D}=M_{11}$.

\subsection{Factorization of mixing matrix}

In general, the lepton mixing matrix can be constructed as

$$
U_{\mathrm{PMNS}}=V_{0} \times U_{1} \times U_{2} \times \ldots,
$$

where different factors come from diagonalization of different mass matrices involved. In our approach we take $V_{0} \sim V_{\mathrm{CKM}}^{\dagger}$. This common part for the quarks and leptons is due to 
the $\mathrm{SO}(10)$ GUT and the CKM physics. The rest, $U_{0} \equiv U_{\mathrm{PMNS}^{0}}=U_{1} \times U_{2} \ldots$, reproduces $U_{\mathrm{TBM}}$ or $U_{\mathrm{BM}}$, i.e. matrices of special type dictated by the flavor symmetry. Here we focus on construction of $U_{0}$ from the double seesaw mechanism, and discuss its TBM or BM forms. The CKM mixing, the non-zero 1-3 mixing, as well as other deviations from the TBM mixing, are probably related to each other and have some other origin, which we refer to as the CKM new physics. In view of small CKM mixing, $U_{0}$ can be considered as the zero-order approximation of the PMNS mixing, $U_{\mathrm{PMNS}^{0}}$.

Flavor features which lead to TBM matrix have been extensively studied before (see $[67,68]$ and references therein). The real TBM matrix can be factorized as

$$
U_{\mathrm{TBM}}=\operatorname{diag}\left(1, \omega^{2}, \omega\right) U_{\omega} U_{13}^{\max T} \operatorname{diag}(1,1, i)
$$

This factorization is not unique: in general any product of $U_{\omega}$ (also with permuted rows and columns) and 2-state maximal mixings, $U_{\omega} U_{12}^{\max }, U_{\omega} U_{13}^{\max }$, or $U_{\omega} U_{23}^{\max }$, leads to the TBM matrix with permuted rows and columns up to rephasing. This, however, does not affect physics: flavor content of the eigenstates with certain eigenvalues is the same for all the cases.

It is straightforward to generalize the factorization via $U_{\omega}$ and $U_{13}^{\max T}$ to

$$
\tilde{U}_{\omega}=D_{\alpha} U_{\omega} U_{A}, \quad \tilde{U}_{13}^{\max T}=U_{A}^{\dagger} U_{13}^{\max T} D_{\beta}
$$

where $U_{A}$ is arbitrary unitary matrix, and $D_{\alpha}$ is arbitrary diagonal phase matrix and the phases can be absorbed into the wave functions of the charged leptons. The matrix $D_{\beta}$ contributes to the Majorana phases of neutrinos. In the generalized form, one may use $U_{A}=\left(U_{12}^{\max T} U_{13}^{\max }\right)^{\dagger}$, so that $U_{\mathrm{PMNS}}=\tilde{U}_{\omega} U_{12}^{\max T}$ and one of the factors is maximal 12 rotation instead of 1-3 rotation. However, now $\tilde{U}_{\omega}=D_{\alpha} U_{\omega} U_{13}^{\max T} U_{12}^{\max }$, which is not reduced to $U_{\omega}$ with permuted columns (rows), and so it is difficult to obtain $U_{\omega}$ as a result of symmetry.

Another possible factorization of $U_{\mathrm{TBM}}$, without using the magic matrix $U_{\omega}$ directly, is $U_{23}^{\max } U_{12}\left(\theta_{\mathrm{TBM}}\right)$, where $\sin ^{2} \theta_{\mathrm{TBM}}=1 / 3$. However, mass matrices diagonalized by $U_{12}\left(\theta_{\text {TBM }}\right)$ do not naturally appear from $A_{4}$ symmetry.

According to the double seesaw, there are three mass matrices relevant for generation of the lepton mixing:

$$
m_{D}, m_{D} M_{D}^{T-1}, M_{S}
$$

where the first matrix is the mass matrix of charged leptons, the second one is the screening factor and the third one is the mass matrix of singlet fermions. In general all these matrices provide contributions to the lepton mixing matrix. Different mass matrices in eq. (2.36) can be responsible for generation of different factors in the TBM matrix. Let us mention some possibilities mostly related to $U_{\omega}$ and $U_{13}^{\max }$, which will be realized in specific schemes in section 3 . 
1. $U_{\omega}$ may follow from the charged leptons, and diagonalize the matrix $m_{D}$, whereas $U_{13}^{\max }$ comes from the neutrino mass matrix, so that

$$
m_{\nu}=\left(\begin{array}{ccc}
x & 0 & z \\
0 & y & 0 \\
z & 0 & x
\end{array}\right) .
$$

In the $\mathrm{SO}(10)$ framework $U_{l}=U_{\omega}^{*}$ implies that $m_{l}=m_{D}=M_{\omega}$ defined in eq. (2.33).

2. $U_{l}=\mathbb{1}$, which means that $m_{D}$ is diagonal, $U_{\omega}$ follows from the portal factor: $m_{D} M_{D}^{T-1} \propto U_{\omega}^{*}$, and $U_{13}^{\max }$ originates from $M_{S}$.

3. One extreme possibility is when $U_{\mathrm{TBM}}$ comes completely from charged leptons, $U_{l}=\left(U_{\mathrm{TBM}}\right)^{\dagger}$. The neutrino diagonalization matrix should be the unit matrix.

4. Another extreme is when the whole $U_{\mathrm{TBM}}$ mixing is generated by $M_{S}$ (i.e. by the hidden sector): $m_{D}=\operatorname{diag}, m_{D}^{T} M_{D}^{-1} \propto \mathbb{1}, M_{S}=M_{\mathrm{TBM}}$. In this case $m_{\nu} \propto M_{S}$.

\subsection{Additional symmetries and VEV alignments}

In what follows we construct schemes for masses and mixing using mass matrices elaborated in this section as building blocks. It is easy to show that in the formulated framework without additional constraints any mass matrix of the light neutrinos can be obtained. Therefore to get certain flavor structures we will use

- minimal possible number flavon fields;

- minimal number of (different) couplings;

- the most symmetric situations when as many as possible couplings are either zero or equal. The latter implies certain extended symmetries and for definiteness we will use additional symmetry $Z_{4}$.

Existence of different energy scales in this framework, $m_{D} \ll M_{D} \ll M_{S}$, allows to realize the idea that different symmetries are realized at different scales with larger symmetry at higher mass scale. Higher scale has larger symmetry, which is then broken explicitly or spontaneously at lower scale. Thus, $M_{S}$ may have additional symmetry which is not realized in portal and visible interactions. Feedback of this breaking onto higher scale physics is expected to be suppressed by factors $M_{D} / M_{S} \sim 10^{-2}$. In particular, due to additional symmetries $M_{S}$ can have the TBM form. Although the symmetry is not realized at the portal scale, the corrections to TBM mixing can be of the order $10^{-2}$. See also [48] for some realizations of this idea.

We formulate conditions on couplings and VEVs which should be satisfied to get the required fermion masses and mixing. We provide some hints of how the conditions can be realized and where they can originate from.

Mechanisms have been elaborated which allow to get relations between VEVs of multiplets. In particular for triplets the alignments $v(1,1,1)$, or $v(0,1,1), v(0,0,1)$ can be 
obtained, as a consequence of symmetry of the potential [69-71]. Usually such potentials have several degenerate vacua, and so some mechanisms of selection should exist. Construction of models which realize the conditions is beyond the scope of this paper.

In various cases we need also correlations of VEVs of singlet and triplet flavons:

$$
h\left(u_{1}, u_{2}, u_{3}\right)^{T}=\sqrt{3} U_{\omega}\left(y v, y^{\prime} v^{\prime}, y^{\prime \prime} v^{\prime \prime}\right)^{T} .
$$

Multiplied by $U_{\omega}^{*}$, the relation can be inverted to:

$$
\left(y v, y^{\prime} v^{\prime}, y^{\prime \prime} v^{\prime \prime}\right)^{T}=h U_{\omega}^{*}\left(u_{1}, u_{2}, u_{3}\right)^{T} / \sqrt{3} .
$$

For equal constants $y^{\prime}=y^{\prime \prime}=y$, it can be written as

$$
\left(v, v^{\prime}, v^{\prime \prime}\right)^{T}=a U_{\omega}^{*}\left(u_{1}, u_{2}, u_{3}\right)^{T},
$$

where $a=h / \sqrt{3} y$.

To reach the correlation (2.38) we introduce a dimensionless auxiliary field $A$ with the VEVs $\langle A\rangle=(a, a, a) / \sqrt{3}$. Then the equality (2.38) is satisfied automatically if

$$
v=\langle(A \cdot \xi)\rangle, \quad v^{\prime}=\left\langle(A \cdot \xi)^{\prime}\right\rangle, \quad v^{\prime \prime}=\left\langle(A \cdot \xi)^{\prime \prime}\right\rangle .
$$

For non-equal constants $\left(y, y^{\prime}, y^{\prime \prime}\right)$ we should substitute $v^{\prime} \rightarrow v^{\prime} y^{\prime} / y$ and $v^{\prime \prime} \rightarrow v^{\prime \prime} y^{\prime \prime} / y$ in the left-hand sides of these equalities. In turn, the relation (2.40) can be induced e.g. by scalar potentials of the form

$$
V(\varphi) \text { eff } \propto-[\varphi-(A \cdot \xi)]^{4}
$$

Consequently, $A \cdot \xi$ should have the same $Z_{4}$ charges as $\varphi$.

\section{Schemes with $S$ transforming as a triplet of $A_{4}$}

For single complete set of the flavons $\xi \sim \mathbf{3}, \vec{\varphi} \sim \overrightarrow{\mathbf{1}}$ the most general $A_{4}$ symmetric Yukawa Lagrangian (at the lowest order) is

$$
\begin{aligned}
\mathcal{L}_{D} & =\frac{10_{H}}{\Lambda_{f}}\left[\vec{y}_{10}\left(16_{F} \cdot 16_{F}\right)_{\varphi} \vec{\varphi}+h_{10}\left(16_{F} \cdot 16_{F} \cdot \xi\right)\right]+y_{10}^{b}\left(16_{F} \cdot 16_{F}\right) 10_{H}, \\
\mathcal{L}_{N S} & =\frac{\overline{16}_{H}}{\Lambda_{f}}\left[\vec{y}_{16}\left(16_{F} \cdot S\right)_{\varphi} \vec{\varphi}+h_{16}\left(16_{F} \cdot S \cdot \xi\right)\right]+y_{16}^{b}\left(16_{F} \cdot S\right) \overline{16}_{H}, \\
\mathcal{L}_{S} & =\vec{y}_{1}(S \cdot S)_{\varphi} \vec{\varphi}+h_{1}(S \cdot S \cdot \xi)+M_{S}^{0}(S \cdot S) .
\end{aligned}
$$

Here

$$
\vec{y}_{10}\left(16_{F} \cdot 16_{F}\right)_{\varphi} \vec{\varphi} \equiv y_{10}\left(16_{F} \cdot 16_{F}\right) \varphi+y_{10}^{\prime}\left(16_{F} \cdot 16_{F}\right)^{\prime \prime} \varphi^{\prime}+y_{10}^{\prime \prime}\left(16_{F} \cdot 16_{F}\right)^{\prime} \varphi^{\prime \prime},
$$

etc., with general definitions of these combinations given in $(2.17)$. The terms $\mathcal{L}_{S}$ contain renormalizable couplings. The symmetry allows also to introduce renormalizable flavonless couplings of $16_{F}$ and $S$. If several sets of flavons $\vec{\varphi}_{a}$ and $\xi_{b}$ exist, for each of them one should introduce interactions similar to those in eq. (3.1). 
All the mass matrices $\left(m_{D}, M_{D}\right.$ and $\left.M_{S}\right)$ generated by this Lagrangian are of the $M_{33}$ type plus matrices proportional to unit matrix. (The latter can be forbidden by additional symmetry.) If the same flavons contribute to all three mass matrices, these matrices are not completely independent being functions of the same set of VEVs. On the other hand, sets of coupling constants for three types of interactions, $\vec{y}_{i} \equiv\left(y_{i}, y_{i}^{\prime}, y_{i}^{\prime \prime}\right)$ and $h_{i}(i=10,16,1)$, can be totally different. With this one can get arbitrary matrices $m_{D}, M_{D}$ and $M_{S}$, and consequently, arbitrary symmetric mass matrix of the light neutrinos.

In what follows we will study the situations that some interactions are forbidden by additional symmetry $Z_{4}$. One possibility is to keep minimal number of couplings in visible and portal sectors, and to obtain all the rest from the hidden sector. Among four types of couplings in the visible sector and portal interactions, $\vec{y}_{10}, h_{10}, \vec{y}_{16}$ and $h_{16}$, at most two can be zero (one for $10_{H}$ and one for $\overline{16}_{H}$ if we do not take into account low-dimension interaction couplings $y^{b}$ ). Next complication is to assume that some couplings among $\vec{y}_{10}$ and $\vec{y}_{16}$ are zero, which would require more symmetries. The couplings of the hidden sector $\vec{y}_{1}$ and $h_{1}$, are also restricted by $Z_{4}$. Thus, we have four possibilities determined by representations of flavons that contribute to the corresponding couplings/mass terms: $h_{10}=h_{16}=0$, which we call the singlet-singlet scheme; $\vec{y}_{10}=\vec{y}_{16}=0$ - the triplet-triplet scheme; $h_{10}=\vec{y}_{16}=0$ - the singlet-triplet scheme; and $\vec{y}_{10}=h_{16}=0$ - the triplet-singlet scheme. We consider these possibilities in order and then present schemes in which both singlets and triplets contribute to the same mass terms.

\subsection{Singlet-singlet flavon scheme}

The scheme with $h_{10}=h_{16}=0$ allows to realize the following scenario:

$$
U_{l}=\mathbb{1}, \quad m_{D} M_{D}^{-1}=\mathbb{1}, \quad U_{S}=U_{\mathrm{TBM}},
$$

where $U_{l}$ diagonalizes $m_{D}$, and $U_{S}$ diagonalizes $M_{S}$. Here the TBM mixing comes from solely the hidden sector. ${ }^{3}$ Only singlet flavons and possibly flavonless operators generate visible and portal masses. The triplet flavon couplings, $h_{10}$ and $h_{16}$, are forbidden by additional $Z_{4}$ symmetry. We introduce flavon singlets $\vec{\varphi}$, which operate in the visible sector and portal terms. One flavon triplet $\xi$ of $A_{4}$ act in the hidden sector. For symmetry assignment under $Z_{4}$ we use

$$
\left\{16_{F}, S\left|10_{H}, \overline{16}_{H}\right| \vec{\varphi}, \xi\right\} \sim\{1,1|i, i|-i, 1\} .
$$

Graphic representation of the scheme is shown in figure 1.

The most general $A_{4} \times Z_{4}$ symmetric Lagrangian has the form

$$
\begin{aligned}
\mathcal{L}_{D} & =\frac{10_{H}}{\Lambda_{f}} \vec{y}_{10}\left(16_{F} \cdot 16_{F}\right)_{\varphi} \vec{\varphi}, \\
\mathcal{L}_{N S} & =\frac{\overline{16}_{H}}{\Lambda_{f}} \vec{y}_{16}\left(16_{F} \cdot S\right)_{\varphi} \vec{\varphi}, \\
\mathcal{L}_{S} & =M_{S}^{0}(S \cdot S)+h_{1}(S \cdot S \cdot \xi) .
\end{aligned}
$$

\footnotetext{
${ }^{3}$ Generation of lepton mixing in the hidden sector has been considered in [48]. The difference is that here we use common non-abelian symmetry in visible and hidden sectors.
} 


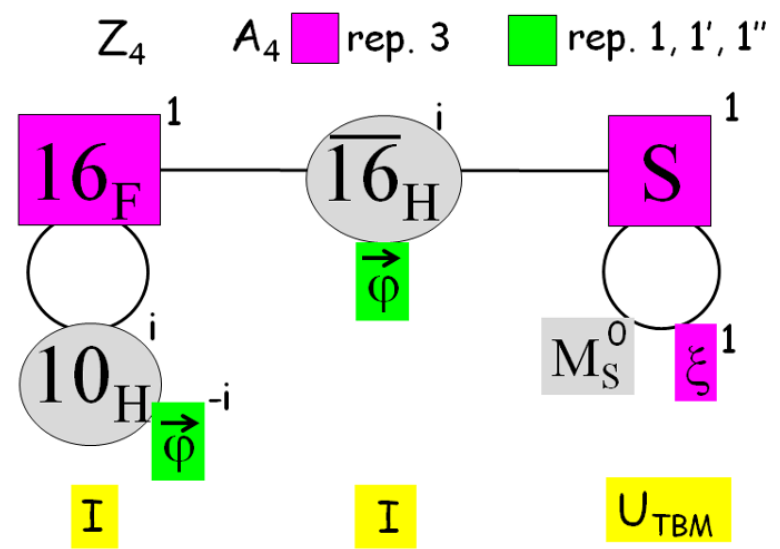

Figure 1. Graphic representation of the singlet-singlet flavon scheme. Numbers at the field notations (i.e. the superscript of each particle content) are the corresponding $Z_{4}$ charges. In the bottom we show rotation matrices generated by the corresponding interactions.

All the mass matrices generated by this Lagrangian are of $M_{33}$ type. Since triplet flavons do not appear in the visible and portal sectors, $m_{D}$ and $M_{D}$ are of $M_{33}^{\text {diag }}$ type (2.19) leading to $U_{l}=\mathbb{1}$. Three eigenvalues of them are arbitrary.

For $M_{D}$ we have an expression similar to $m_{D}$ with substitutions $\vec{y}_{10} \leftrightarrow \vec{y}_{16}$ and $10_{H} \leftrightarrow \overline{16}_{H}$. Complete screening requires proportionality (correlation) of the couplings constants of the Dirac and portal interactions, $\vec{y}_{10}$ and $\vec{y}_{16}$ :

$$
y_{10}: y_{10}^{\prime}: y_{10}^{\prime \prime}=y_{16}: y_{16}^{\prime}: y_{16}^{\prime \prime} .
$$

This is enough for the screening since the same flavon fields contribute to both mass matrices. The proportionality of eq. (3.3) is rather a generic requirement for several schemes, so we discuss it in some details. This proportionality can be obtained in various ways as follows:

(i) All the coupling constants for $10_{H}$ and independently $\overline{16}_{H}$ are equal to each other (or approximately equal):

$$
y_{10}=y_{10}^{\prime}=y_{10}^{\prime \prime}, \quad y_{16}=y_{16}^{\prime}=y_{16}^{\prime \prime} .
$$

In this case correlations are not needed. The equalities can be residuals of embedding of $A_{4}$ into $\mathrm{SO}(3)$, so that the singlet of $A_{4}\left(1,1^{\prime}, 1^{\prime \prime}\right)$ steam from the triplet representation of $\mathrm{SO}(3)$. The (approximate) equality of the coupling constants is also needed to obtain the hierarchy of masses of quarks and charged leptons.

(ii) The proportionality can be a consequence of further unification of $16_{F}$ and $S$ in 27-plets of $E_{6}$.

In both cases (i) and (ii) the $Z_{4}$ charges of $16_{F}$ and $S$ should be equal, as given in eq. (3.2). 
(iii) The proportionality can be a consequence of symmetry with respect to permutation $16_{F} \leftrightarrow S$ and $10_{H} \leftrightarrow \overline{16}_{H}$. In this case also $Z_{4}$ charges of $10_{H}$ and $\overline{16}_{H}$ should be the same, which is satisfied in the symmetry assignment.

Under condition (3.3) the matrices $m_{D}$ and $M_{D}$ have the same flavor structure, so that $m_{D} / M_{D} \propto \mathbb{1}$.

Due to the absence of singlet flavons, $M_{S}$ should have all equal diagonal elements from the bare mass terms. The VEV alignment of the triplet $\langle\xi\rangle=u(1,1,1)$ leads to the matrix $M_{S}$ in the form

$$
M_{S}=M_{33}^{\text {special }}+\Delta M_{S}
$$

where the special matrix $M_{33}^{\text {special }}$ is given in (2.24) with $\mu=M_{S}^{0}$ and $\beta=h_{1} u$. A perturbation matrix $\Delta M_{S}$ is needed, and should be of the form $\Delta M$ in eq. (2.26), so that the total $M_{S}$ has the TBM form and thus is diagonalized by $U_{\text {TBM }}$. The eigenvalues of $M_{S}$ equal

$$
\lambda_{1,3}=M_{S}^{0}-h_{1} u \pm M_{0}, \quad \lambda_{2}=M_{S}^{0}+2 h_{1} u+M_{0}
$$

and there is enough freedom to get any spectrum. ${ }^{4}$ The light neutrino masses satisfy that $m_{i} \propto \lambda_{i}$.

There are several ways to obtain the additional correction $\Delta M_{S}$ in the form of $\Delta M$ :

1. A correction proportional to $\Delta M$ but directly contributing to $m_{\nu}$ can come from additional fermion singlets $S, S^{\prime}, S^{\prime \prime}$, as we will discuss in section 5.2.

2. Another modification of the scheme which would be equivalent to the correction $\Delta M(2.26)$ is to introduce flavons $\phi, \phi^{\prime}, \phi^{\prime \prime}$ which modify the diagonal elements of $M_{S}$. Suppose that $\phi, \phi^{\prime}, \phi^{\prime \prime}$ acquire VEVs $\left(v_{1}, v_{1}^{\prime}, v_{1}^{\prime \prime}\right)$ which satisfy together with couplings the equality $y_{1} v_{1}=y_{1}^{\prime} v_{1}^{\prime}=y_{1}^{\prime \prime} v_{1}^{\prime \prime}$. And differently from above, $\langle\xi\rangle=\left(u_{1}, u_{2}, u_{3}\right)$. Then the mass matrix of singlets can have the form

$$
M_{S}=h_{1}\left(\begin{array}{ccc}
3 y_{1} v_{1} / h_{1} & u_{3} & u_{2} \\
\ldots & 0 & u_{1} \\
\ldots & \ldots & 0
\end{array}\right)+M_{S}^{0} \mathbb{1}
$$

In the case of complete screening it gives the light neutrino mass matrix: $m_{\nu}=b \cdot M_{S}$. Imposing further equality $u_{3} \equiv u_{2}$ we obtain from (3.4) maximal 2-3 mixing, and still enough freedom is left to fit all the data. In particular, equality $h_{1} u_{1}=h_{1} u_{2}+3 y_{1} v_{1}$ leads to the TBM mixing and to the mass eigenvalues

$$
m_{1}=b\left(M_{S}^{0}+h_{1} u_{1}-2 h_{1} u_{2}\right), \quad m_{2}=b\left(M_{S}^{0}+h_{1} u_{1}+h_{1} u_{2}\right), \quad m_{3}=b\left(M_{S}^{0}-h_{1} u_{1}\right),
$$

which can reproduce essentially any neutrino mass spectrum, and therefore normal or inverted mass hierarchy.

\footnotetext{
${ }^{4}$ Notice that $M_{0} \neq 0$ is important, otherwise we would have two degenerate values $\lambda_{1,3}=M_{S}^{0}-h_{1} u$. That would lead to $m_{1}=m_{3}$ for light neutrinos, which contradicts observations.
} 


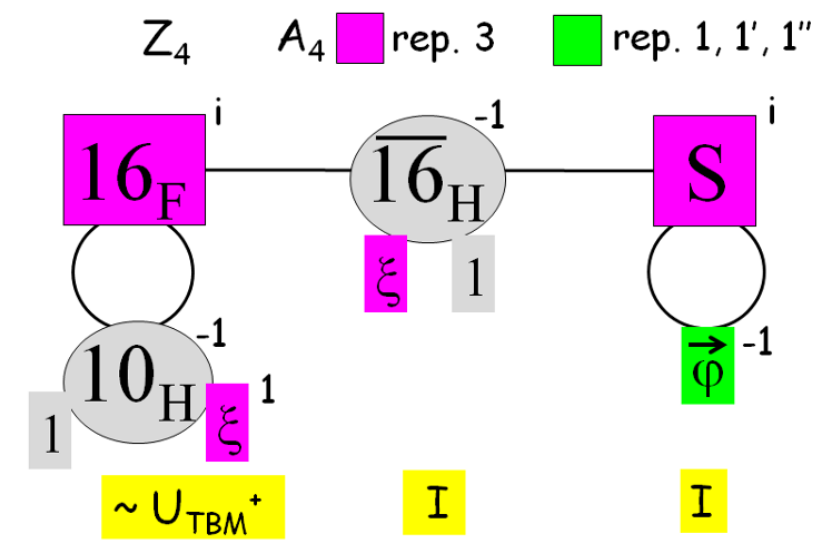

Figure 2. The same as in figure 1, but for the triplet-triplet flavon scheme. Grey box with 1 corresponds to flavonless couplings.

\subsection{Triplet-triplet flavon scheme}

The Lagrangian is given in eq. (3.1) with $\vec{y}_{10}=\vec{y}_{16}=0$ and graphic representation of the scheme is shown in figure 2 . It allows to realize a scenario

$$
U_{l} \approx U_{\mathrm{TBM}}^{\dagger}, \quad m_{D} / M_{D} \propto \mathbb{1}, \quad U_{S}=\mathbb{1},
$$

in which the mixing originates from the charged leptons. Vanishing singlet couplings $\vec{y}_{10}$ and $\vec{y}_{16}$ can be obtained by imposing the $Z_{4}$ assignment

$$
\left\{16_{F}, S\left|10_{H}, \overline{16}_{H}\right| \vec{\varphi}, \xi\right\} \sim\{i, i|-1,-1|-1,1\} .
$$

Now only the triplet and operators without flavons contribute to the visible and portal masses. Therefore

$$
m_{D}=m_{D}^{0} \cdot \mathbb{1}+r M_{33}^{\xi}, \quad M_{D}=M_{D}^{0} \cdot \mathbb{1}+M_{33}^{\xi},
$$

where $m_{D}^{0}=y_{10}^{b}\left\langle 10_{H}\right\rangle, m_{D}^{0}=y_{16}^{b}\left\langle\overline{16}_{H}\right\rangle$ and $r \equiv h_{10}\left\langle 10_{H}\right\rangle / h_{16}\left\langle\overline{16}_{H}\right\rangle$.

Diagonalizing $m_{D}$ we find that masses of charged leptons can be reproduced if

$$
\left|m_{D}^{0}\right|=\frac{1}{3}\left(m_{e}+m_{\mu}+m_{\tau}\right), \quad\left(h_{10}\right)^{2} \sum_{i} u_{i}^{2} \approx \frac{1}{3} m_{\tau}^{2}, \quad\left(h_{10}\right)^{3} u_{1} u_{2} u_{3} \approx \frac{1}{27} m_{\tau}^{3} .
$$

The second and third conditions are satisfied if, e.g. $u_{1} \approx u_{2} \approx u_{3} \approx m_{\tau} /\left(3 h_{10}\right)$.

One important feature of this case is that the matrices $m_{D}$ and $M_{D}$ in eq. (3.6) have different eigenvalues but the same mixing. Since off-diagonal parts of both matrices are generated by the same flavon field $\xi$, they are diagonalized by the same rotation $U_{33}$ which diagonalizes $M_{33}^{\xi}: U_{33}^{T} M_{33}^{\xi} U_{33}=M_{D}^{\text {diag }}$ (see appendix C). At the same time, depending on relative size of diagonal and off-diagonal contributions, the matrices $m_{D}$ and $M_{D}$ can have different hierarchies of eigenvalues. Consequently, $U_{l}=U_{33}$ and in the flavor basis the mass matrix of light neutrinos becomes

$$
m_{\nu}=m_{D}^{\text {diag }}\left(M_{D}^{\text {diag }}\right)^{-1} U_{33}^{T} M_{S} U_{33}\left(M_{D}^{\text {diag }}\right)^{-1} m_{D}^{\text {diag }} .
$$


Complete screening, $m_{D}\left(M_{D}\right)^{-1}=c \cdot \mathbb{1}$, requires that $y_{10}^{b}: y_{16}^{b}=h_{10}: h_{16}$. In this case we obtain from eq. (3.8)

$$
m_{\nu}=c^{2} U_{33}^{T} M_{S} U_{33}=c^{2} U_{l}^{T} M_{S} U_{l}
$$

in the flavor basis.

In the hidden sector due to $Z_{4}$ symmetry given by eq. (3.5) $h_{1}=0$, and so the flavon triplet does not contribute to the singlet mass matrix. Therefore $M_{S}=M_{33}^{\varphi}$ and the neutrino matrix in eq. (3.9) is diagonalized by $U_{33}^{\dagger}$. Consequently, the neutrino mixing $U_{0}=U_{33}^{\dagger}$. This result can be immediately obtained by noticing that in the original symmetry basis and in the presence of complete screening the neutrino mass matrix is diagonal whereas the lepton mass matrix is diagonalized by $U_{l}=U_{33}$.

In turn, the matrix $U_{33}$ can reproduce the TBM or BM mixing. Indeed, if the 13 mixing is generated by the CKM physics, the 1-3 mixing, which originates from $U_{33}$, should be zero. So, in terms of the standard parametrization of $U_{\text {PMNS }}$ we have to have $U_{33}=U_{12}\left(\theta_{12}\right)^{T} U_{23}\left(\theta_{23}\right)^{T}$. This requires equality $u_{2}=u_{1}$ in $M_{33}^{\xi}(2.23)$ which leads to the maximal 1-2 mixing, $\theta_{12}=\pi / 4$. Then for the $2-3$ mixing we obtain

$$
\tan 2 \theta_{23}=\frac{2 \sqrt{2} u_{1}}{u_{3}}
$$

For $u_{1} \gg u_{3}$ this equation would give nearly maximal 2-3 mixing. However, this is not consistent with (3.7) which has for $u_{2}=u_{1}$ two solutions: $u_{3}=u_{1}$ and $u_{3}=(2 \sqrt{2} i) u_{1}$. In the first (best) case we obtain $\theta_{23}=35^{\circ}$, which can be slightly bigger if $u_{2} \neq u_{1}$, but the latter corresponds to non-zero 1-3 mixing. Thus, the scheme produces the bi-large mixing from the neutrino sector. Neutrino masses are given by eigenvalues of $M_{33}^{\varphi}$. According to the discussion in section 2.4 (see eq. (2.19) there and also appendix B) any type of neutrino mass spectrum and any hierarchy can be obtained, depending on the Yukawa couplings and VEVs of $\vec{\varphi}$.

\subsection{Singlet-triplet flavon scheme}

The Lagrangian of this scheme is given by eq. (3.1) with $h_{10}=\vec{y}_{16}=0$, and the graphic representation is shown in figure 3. Only flavon singlets contribute to the Dirac mass matrix and only triplet flavons to the portal mass matrix. Here we can realize the scenario

$$
U_{l}=\mathbb{1}, \quad M_{D} \propto M_{S}=M_{33}^{\xi}
$$

with cancellation of the portal and hidden sector matrices in the double seesaw mechanism. Vanishing $h_{10}$ and $\vec{y}_{16}$ can be obtained with $Z_{4}$ symmetry assignment

$$
\left\{16_{F}, S\left|10_{H}, \overline{16}_{H}\right| \vec{\varphi}, \xi\right\} \sim\{i,-i|-1,-1| 1,-1\} .
$$

The key feature is that $16_{F}$ and $S$ have different $Z_{4}$ charges. The $Z_{4}$ symmetry also forbids the low-dimension (flavonless) contribution of the mass $M_{D}^{0}$, so that

$$
m_{D}=m_{D}^{0} \mathbb{1}+M_{33}^{\varphi}, \quad M_{D}=M_{33}^{\xi},
$$




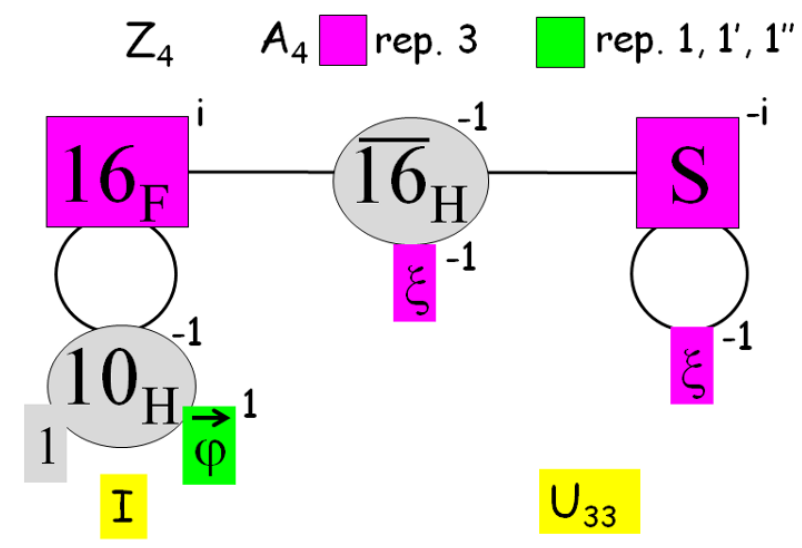

Figure 3. The same as in figure 1, but for the singlet-triplet flavon scheme. The matrix which diagonalize $m_{\nu}$ comes from a non-trivial combination of $U_{33}$ and $m_{D}^{\text {diag }}$ (see the text).

where $m_{D}^{0}=y^{b}\left\langle 10_{H}\right\rangle$. The matrix $m_{D}$ has enough free parameters to reproduce the charged lepton masses.

With the assignment in figure 3 , only triplet $\xi$ contributes to the hidden sector matrix, so that $M_{S} \propto M_{33}^{\xi}$. Furthermore, since the same $\xi$ contributes to both $M_{S}$ and $M_{D}$ we have $M_{S} \propto M_{D}$. Therefore $M_{S}$ cancels with one inverse $M_{D}$ in the double seesaw formula. As a result, the light neutrino mass matrix becomes

$$
m_{\nu} \propto m_{l}^{\operatorname{diag}}\left(M_{33}^{\xi}\right)^{-1} m_{l}^{\text {diag }} .
$$

Effectively the expression for mass looks like a single seesaw and only the overall scale of the $\mathrm{RH}$ neutrino masses imprints information about the presence of the hidden sector.

In spite of strong hierarchy in $m_{l}^{\text {diag }}$ the neutrino mass matrix in (3.11) can be made in agreement with data due to the special form of $M_{33}^{\xi}$, such that its inverse matrix,

$$
\left(M_{33}^{\xi}\right)^{-1}=\frac{1}{2 h u_{1} u_{2} u_{3}}\left(\begin{array}{ccc}
-u_{1}^{2} & u_{1} u_{2} & u_{1} u_{3} \\
\ldots & -u_{2}^{2} & u_{2} u_{3} \\
\ldots & \ldots & -u_{3}^{2}
\end{array}\right),
$$

has quadratic hierarchy in $u_{i}$. To show this we take $m_{l}^{\operatorname{diag}}=m_{\tau} \operatorname{diag}\left(\lambda^{6}, \lambda^{2}, 1\right)$, where $\lambda \equiv \sin \theta_{C} \simeq 0.22$ is the Cabibbo angle. Then for VEV the hierarchy $\langle\xi\rangle=\left(u_{1}, u_{2}, u_{3}\right)=$ $\left(-1, \lambda^{3}, \lambda^{5}\right)$ with coefficients of the order 1 (which is even weaker than the hierarchy in $\left.m_{l}^{\text {diag }}\right)$ eqs. (3.11) and (3.12) lead to

$$
m_{\nu} \propto\left(\begin{array}{ccc}
-\lambda^{4} & \lambda^{3} & \lambda^{3} \\
\ldots & \lambda^{2} & -\lambda^{2} \\
\ldots & \ldots & \lambda^{2}
\end{array}\right)
$$

This matrix produces a zero (small) $\theta_{13}$, maximal 2-3 mixing and large 1-2 mixing. It only corresponds to the normal mass hierarchy, which is due to the connection of neutrino masses with the mass hierarchy of charged fermions. 


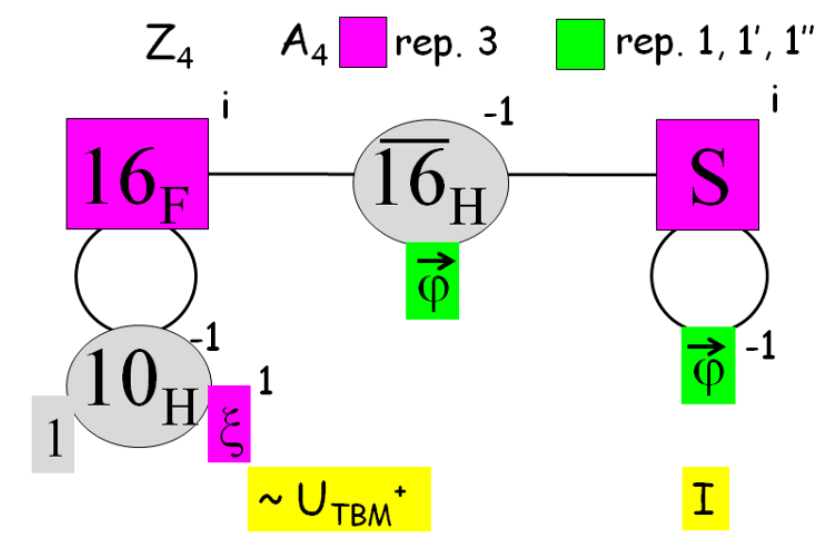

Figure 4. The same as in figure 1, but for the triplet-singlet flavon scheme.

\subsection{Triplet-singlet flavon scheme}

This case is in some sense opposite to the previous one: the triplet $\xi$ produces the Dirac mass matrix, whereas singlets generate the portal mass matrix, see figure 4 . The Lagrangian is given by eq. (3.1) with $\vec{y}_{10}=h_{16}=0$. Vanishing $\vec{y}_{10}$ and $h_{16}$ can be obtained by the $Z_{4}$ with assignment

$$
\left\{16_{F}, S\left|10_{H}, \overline{16}_{H}\right| \vec{\varphi}, \xi\right\} \sim\{i,-i|-1,-1|-1,1\}
$$

It leads to

$$
m_{D}=m_{D}^{0} \cdot \mathbb{1}+M_{33}^{\xi}, \quad M_{D}=M_{33}^{\varphi} .
$$

Symmetry allows flavonless contribution $m_{D}^{0}$, whereas a similar contribution to $M_{D}$ is forbidden. Generation of the Dirac mass matrix $m_{D}$ is similar to the case in section 3.2.

The scheme allows to realize an interesting possibility when mixing comes essentially from the $\mathrm{RH}$ rotations which diagonalize the neutrino Dirac mass matrix. This is possible due to the Majorana nature of neutrinos. If $M_{D} \propto \mathbb{1}$ (only the true singlet, $\varphi$, contributes), then

$$
m_{\nu} \propto m_{D}^{\operatorname{diag}} \cdot U_{33}^{\dagger} M_{S} U_{33}^{*} \cdot m_{D}^{\text {diag }}
$$

in the flavor basis, and $m_{D}^{\text {diag }}=m_{l}^{\text {diag }}$.

Due to $Z_{4}$ only $\vec{\varphi}$ can couple to $S$, and consequently, $M_{S}$ is diagonal. Even in this case by selecting parameters of $U_{33}$ one can reproduce observables. Note that due to the hierarchical structure of $m_{l}^{\text {diag }}$, normal mass hierarchy of SM neutrinos is preferred here, similarly to the case of section 3.3.

\subsection{Scheme with mixed contributions: the TBM mixing}

Here and in the next subsection we consider general situations when both triplet and singlet flavons interact in the visible sector. The model in this subsection realizes the scenario in which

$$
U_{l}=U_{\omega}^{*}, \quad m_{D}^{T} M_{D}^{-1} \propto \mathbb{1}, \quad U_{S}=U_{13}^{\max } .
$$




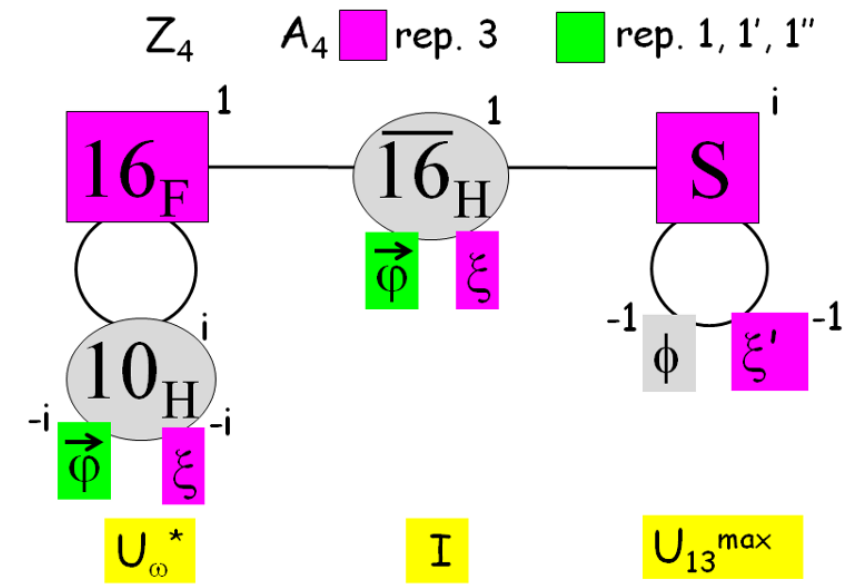

Figure 5. The same as in figure 1 but for the mixed flavon scheme for the TBM mixing.

So, $U_{\omega}$ appears from the charged leptons mass matrix whereas the hidden sector gives maximal 1-3 mixing, and there is a complete screening. As a result, $U_{0} \propto U_{\omega} U_{13}^{\max }=U_{\mathrm{TBM}}$.

The symmetry assignments of the field content we use is

$$
\left\{16_{F}, S\left|10_{H}, \overline{16}_{H}\right| \vec{\varphi}, \phi, \xi, \xi^{\prime}\right\} \sim\{1, i|i, 1|-i,-1,-i,-1\}
$$

under $Z_{4}$, and the full graphic representation are shown in figure 5 .

Both the singlet $\vec{\varphi}$ and triplet $\xi$ flavons, with arbitrary VEVs, participate in visible and portal interactions. The others, $\phi$ and $\xi^{\prime}$, exclusively couple with fermion singlets.

The Lagrangian has the following terms

$$
\begin{aligned}
\mathcal{L}_{D} & =\frac{10_{H}}{\Lambda_{f}}\left[\vec{y}_{10}\left(16_{F} \cdot 16_{F}\right)_{\varphi} \vec{\varphi}+h_{10}\left(16_{F} \cdot 16_{F} \cdot \xi\right)\right], \\
\mathcal{L}_{N S} & =\frac{\overline{16}_{H}}{\Lambda_{f}}\left[\vec{y}_{16}\left(16_{F} \cdot S\right)_{\varphi} \vec{\varphi}+h_{16}\left(16_{F} \cdot S \cdot \xi\right)\right], \\
\mathcal{L}_{S} & =y_{1}(S \cdot S) \phi+h_{1}^{\prime}\left(S \cdot S \cdot \xi^{\prime}\right) .
\end{aligned}
$$

The $Z_{4}$ symmetry forbids all the interactions without flavons including the bare mass terms.

For the Dirac mass matrix we obtain

$$
m_{D}=\frac{\left\langle 10_{H}\right\rangle}{\Lambda_{f}}\left(\begin{array}{ccc}
M_{1} & h_{10} u_{3} & h_{10} u_{2} \\
\ldots & M_{2} & h_{10} u_{1} \\
\ldots & \ldots & M_{3}
\end{array}\right),
$$

where $M_{i}$ are defined in eq. (2.20). To be diagonalized by $U_{\omega}$ this matrix should be of the form eq. (2.33). This requires that $M_{i}=h_{10} u_{i}$, or according to eq. (2.20), the relation (2.38) with $h=h_{10}$ and $\vec{y}=\vec{y}_{10}$.

Using this VEV relation, the mass matrix can be written as

$$
m_{D}=\frac{h_{10}\left\langle 10_{H}\right\rangle}{\Lambda_{f}}\left(\begin{array}{lll}
u_{1} & u_{3} & u_{2} \\
\ldots & u_{2} & u_{1} \\
\ldots & \ldots & u_{3}
\end{array}\right) .
$$


It is diagonalized as $U_{l}^{\dagger} m_{D} V_{R}$, where $U_{l}=U_{\omega}^{*}$ and $V_{R}=U_{\omega}$. The eigenvalues of the matrix equal

$$
\left(m_{D 1}, m_{D 2}, m_{D 3}\right)^{T}=\frac{h_{10}\left\langle 10_{H}\right\rangle}{\Lambda_{f}}\left(\sqrt{3} U_{\omega}^{*}\right)\left(u_{1}, u_{2}, u_{3}\right)^{T}=\frac{3\left\langle 10_{H}\right\rangle}{\Lambda_{f}}\left(y_{10} v, y_{10}^{\prime} v^{\prime}, y_{10}^{\prime \prime} v^{\prime \prime}\right)^{T},
$$

so the mass hierarchy requires that $y_{10} v \ll y_{10}^{\prime} v^{\prime} \ll y_{10}^{\prime \prime} v^{\prime \prime}$.

The same flavons $\vec{\varphi}$ and $\xi$ appear in $\mathcal{L}_{D}$ and $\mathcal{L}_{N S}$. Therefore, the flavor structures of mass matrices $m_{D}$ and $M_{D}$ are the same provided that the Yukawa couplings of $10_{H}$ and $16_{H}$ are proportional to each other

$$
y_{10}: y_{10}^{\prime}: y_{10}^{\prime \prime}: h_{10}=y_{16}: y_{16}^{\prime}: y_{16}^{\prime \prime}: h_{16}
$$

That is, now couplings of $\vec{\varphi}$ and $\xi$ should also correlate. Possible origins of this proportionality have been discussed in section 3.1. As a consequence,

$$
m_{D}^{\nu}=m_{l}=m_{D}=\frac{y_{10}\left\langle 10_{H}\right\rangle}{y_{16}\left\langle\overline{16}_{H}\right\rangle} M_{D}
$$

and therefore $m_{D} M_{D}^{-1 T}=y_{10}\left\langle 10_{H}\right\rangle /\left(y_{16}\left\langle\overline{16}_{H}\right\rangle\right) \cdot \mathbb{1}$, reproducing the complete screening.

Maximal 1-3 mixing should be obtained from $M_{S}$ in the form of $M_{33}$. If $\langle\phi\rangle=v_{1}$ and the VEV of the triplet is aligned as $\left\langle\xi^{\prime}\right\rangle \rightarrow\left(0, u^{\prime}, 0\right)$, we obtain

$$
M_{S}=\left(\begin{array}{ccc}
y_{1} v_{1} & 0 & h_{1}^{\prime} u^{\prime} \\
\ldots & y_{1} v_{1} & 0 \\
\ldots & \ldots & y_{1} v_{1}
\end{array}\right)
$$

The eigenvalues of $M_{S}$ equal $y_{1} v_{1} \pm h^{\prime} u^{\prime}$ and $y_{1} v_{1}$, and consequently, the light neutrino masses equal

$$
m_{1,3}=B\left(y_{1} v_{1} \pm h_{1}^{\prime} u^{\prime}\right), \quad m_{2}=B y_{1} v_{1}, \quad B \equiv \frac{y_{10}^{2}\left\langle 10_{H}\right\rangle^{2}}{y_{16}^{2}\left\langle\overline{16}_{H}\right\rangle^{2}} .
$$

This gives normal mass ordering with $m_{1}: m_{2}: m_{3} \simeq 1: 1: 3$, if $h_{1}^{\prime} u^{\prime} \sim-2 y_{1} v_{1}$. The inverted mass ordering can be obtained if two additional flavon singlets, $\phi^{\prime}$ and $\phi^{\prime \prime}$, are introduced in the hidden sector with couplings satisfying $y_{1}^{\prime}\left\langle\phi^{\prime}\right\rangle=\omega y_{1}^{\prime \prime}\left\langle\phi^{\prime \prime}\right\rangle$. This adds one free parameter to $M_{S}$, and thus, allows arbitrary neutrino masses without changing maximal 1-3 mixing.

The model can be simplified if certain high-order corrections to $m_{D}$ are added. Assume that $\left\langle\varphi^{\prime}\right\rangle=\left\langle\varphi^{\prime \prime}\right\rangle=0$, or simply that $\varphi^{\prime}$ and $\varphi^{\prime \prime}$ are removed from the scheme. In practice, this is equivalent to the case $\vec{y}_{10}=0$ but $m_{D}^{0} \neq 0$. If then

$$
\langle\xi\rangle=u(1,1,1)
$$

we obtain

$$
m_{D}=M_{33}^{\text {special }}
$$

of eq. (2.24) with $\mu=y_{10} v$ and $\beta=h_{10} u$. The equality (3.15) and $M_{S}$ remain unchanged. 
To break the degeneracy of eigenvalues of $m_{D}$ and fix $U_{l}=U_{\omega}$, one has to introduce a non-symmetric correction $\delta m_{D}$ (see e.g. $\Delta M^{\prime}$ in eq. (2.26)). In particular, one can use the anti-symmetric correction

$$
\delta m_{D}=\delta m\left(\begin{array}{ccc}
0 & 1 & -1 \\
-1 & 0 & 1 \\
1 & -1 & 0
\end{array}\right)
$$

which originates from the effective $120_{H}$. The latter appears from non-renormalizable interactions

$$
\frac{1}{\Lambda} 16_{F} 16_{F} 16_{H} 16_{H}, \quad 16_{F} 16_{F} 45_{H} 10_{H},
$$

noticing that the decomposition of $\left(16_{H} \times 16_{H}\right)$ contains $120_{H}$, see $[29,72,73]$ for examples. As a result, $U_{\mathrm{PMNS}}=U_{\omega} U_{13}^{\max }$ with non-degenerate masses of charged leptons given by

$$
m_{D 1}=y_{10} v+2 h_{10} u, \quad m_{D 2, D 3}=y_{10} v-h_{10} u \mp i \sqrt{3} \delta m .
$$

To keep screening, similar corrections should also be introduced for $M_{D}$. It is straightforward to see that the masses of light neutrinos are the same as in eq. (3.16), because $M_{S}$ is unchanged.

\subsection{Scheme with mixed contributions: the BM mixing}

Our framework also allows for new possibilities to produce the BM mixing (see appendix D). The BM mixing can be factorized by two maximal mixing rotations: $U_{\mathrm{BM}}=U_{23}^{\max } U_{12}^{\max }$. In this connection we present a scenario in which maximal 2-3 rotation comes from the charged leptons and the maximal 1-2 one arises from the hidden sector:

$$
U_{l}=\left(U_{23}^{\max }\right)^{T}, \quad m_{D}\left(M_{D}\right)^{-1} \propto \mathbb{1}, \quad U_{S}=U_{12}^{\max } .
$$

The particle content and the charge assignments are given in figure 6 , where the $Z_{4}$ charges are

$$
\left\{16_{F}, S\left|10_{H}, \overline{16}_{H}\right| \vec{\varphi}, \phi, \xi, \xi^{\prime}\right\} \sim\{1, i|-i,-1| i,-1, i,-1\},
$$

respectively. All the mass matrices are of the type $M_{33}$. Bare mass terms and operators without flavons are forbidden by $Z_{4}$ symmetry. The symmetry assignment allows flavon singlets $\vec{\varphi}$ and triplet $\xi$ to couple with fermions in visible and portal interactions, while the other two flavons, singlet $\phi$ and triplet $\xi^{\prime}$, appear in the hidden sector only.

To get maximal 2-3 mixing from the charged leptons, where $m_{l}=m_{D}$, we impose equalities

$$
y_{10}^{\prime}=y_{10}^{\prime \prime}, \quad v^{\prime}=v^{\prime \prime}, \quad\langle\xi\rangle=(u, 0,0) .
$$

These equalities imply the permutation symmetry $\left({ }^{\prime} \leftrightarrow{ }^{\prime \prime}\right)$ in the visible and portal sectors, and therefore lead to maximal 2-3 mixing. Under conditions (3.17) we obtain

$$
m_{D}=\frac{\left\langle 10_{H}\right\rangle}{\Lambda_{f}}\left(\begin{array}{ccc}
y_{10} v+2 y_{10}^{\prime} v^{\prime} & 0 & 0 \\
0 & y_{10} v-y_{10}^{\prime} v^{\prime} & h_{10} u \\
0 & h_{10} u & y_{10} v-y_{10}^{\prime} v^{\prime}
\end{array}\right)
$$




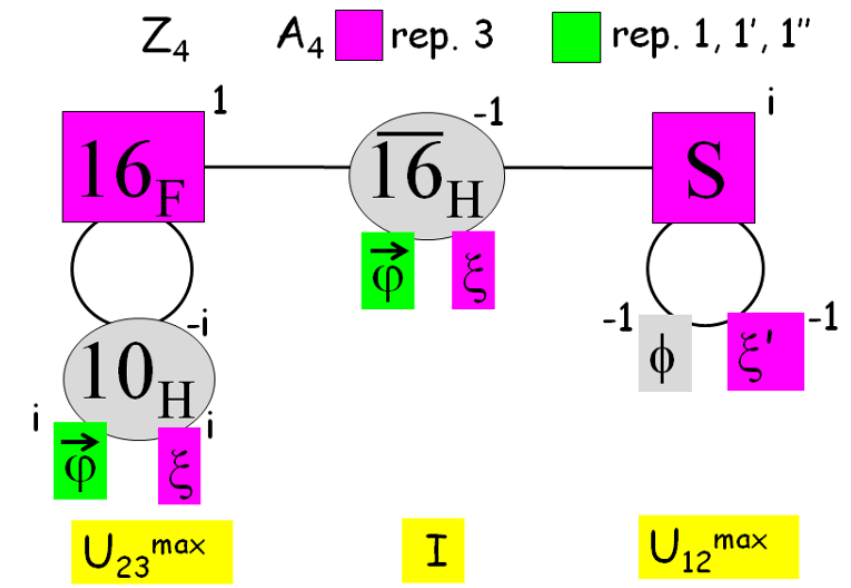

Figure 6. The same as in figure 1 but for the mixed flavon scheme for the BM mixing.

The eigenvalues of this matrix equal

$$
m_{D 1}=\frac{\left\langle 10_{H}\right\rangle}{\Lambda_{f}}\left(y_{10} v+2 y_{10}^{\prime} v^{\prime}\right), m_{D 2, D 3}=\frac{\left\langle 10_{H}\right\rangle}{\Lambda_{f}}\left(y_{10} v-y_{10}^{\prime} v^{\prime} \mp h_{10} u\right) .
$$

To obtain mass hierarchy we should arrange that $y_{10}^{\prime} v^{\prime} \approx-0.5 y_{10} v$, which leads to

$$
m_{D 2} \simeq \frac{\left\langle 10_{H}\right\rangle}{\Lambda_{f}}\left(\frac{3}{2} y_{10} v-h_{10} u\right), \quad m_{D 3} \simeq \frac{\left\langle 10_{H}\right\rangle}{\Lambda_{f}}\left(\frac{3}{2} y_{10} v+h_{10} u\right)
$$

and $m_{D 1} \ll m_{D 2}$. Additional relation $h_{10} u \approx \frac{3}{2} y_{10} v$ gives $m_{D 2} \ll m_{D 3}$.

To realize complete screening, $m_{D} \propto M_{D}$, we impose the proportionality of both singlet and triplet couplings $\vec{y}$ and $h$ with $10_{H}$-plets and $\overline{16}_{H}$-plets as eq. (3.14).

The maximal 1-2 mixing from $M_{S}$ requires that the second flavon triplet, interacting in the hidden sector, has $\mathrm{VEV}\left\langle\xi^{\prime}\right\rangle \rightarrow\left(0,0, u^{\prime}\right)$, which implies the permutation symmetry of the first and second generations in hidden sector. Equal diagonal elements of $M_{S}$ are generated by singlet $\phi$ with $\operatorname{VEV}\langle\phi\rangle=v_{1}$. Under these conditions

$$
M_{S}=\left(\begin{array}{ccc}
y_{1} v_{1} & h_{1}^{\prime} u^{\prime} & 0 \\
h_{1}^{\prime} u^{\prime} & y_{1} v_{1} & 0 \\
0 & 0 & y_{1} v_{1}
\end{array}\right) .
$$

Since $m_{\nu} \propto M_{S}$, an agreement with observed mass spectrum can be achieved for the inverted mass hierarchy when $\left|y_{1} v_{1}\right| \ll\left|h_{1}^{\prime} u^{\prime}\right|$. As a result, the three light neutrino masses equal $m_{3} \propto y_{1} v_{1}, m_{2} \approx m_{1} \propto h^{\prime} u^{\prime}$ and $\Delta m_{21}^{2} \propto 4 y_{1} v_{1} h_{1}^{\prime} u^{\prime}$.

To obtain normal mass hierarchy we can introduce also $\phi^{\prime}$ and $\phi^{\prime \prime}$ with couplings $y_{1}^{\prime}\left\langle\phi^{\prime}\right\rangle=\omega y_{1}^{\prime \prime}\left\langle\phi^{\prime \prime}\right\rangle$, which actually allows to obtain arbitrary values of neutrino masses. Recall that conditions we impose here can be consequences of additional symmetries operating in the hidden sector. 


\section{Schemes with $S$ transforming as singlets of $A_{4}$}

For $\vec{S}=\left(S, S^{\prime}, S^{\prime \prime}\right) \sim\left(\mathbf{1}, \mathbf{1}^{\prime}, \mathbf{1}^{\prime \prime}\right)$ the portal and the hidden parts of the Lagrangian change with respect to eq. (3.1):

$$
\begin{aligned}
\mathcal{L}_{N S}= & \frac{\overline{16}_{H}}{\Lambda_{f}} \vec{h}_{16}\left(16_{F} \cdot \xi\right)_{S} \vec{S} \\
\mathcal{L}_{S}= & M_{S}^{0} S S+2 \tilde{M}_{S}^{0} S^{\prime} S^{\prime \prime}+\left(y_{11} S S+2 y_{23} S^{\prime} S^{\prime \prime}\right) \varphi \\
& +\left(y_{22} S^{\prime} S^{\prime}+2 y_{13} S S^{\prime \prime}\right) \varphi^{\prime}+\left(2 y_{12} S S^{\prime}+y_{33} S^{\prime \prime} S^{\prime \prime}\right) \varphi^{\prime \prime} .
\end{aligned}
$$

The visible interactions are the same as before, so that the Dirac mass matrices have two contributions: one from singlets $\vec{\varphi}$ and the other from triplet $\xi$ of $A_{4}$. Since the fermion singlets $S$ transform as the three one-dimensional representations of $A_{4}$, the portal term has only one contribution: from triplet $\xi$. Therefore, in contrast to the schemes in the previous section with $S \sim \mathbf{3}$, now we can only have two possibilities for zero couplings related to the Dirac terms: $h_{10}=0$ or $\vec{y}_{10}=0$. For both possibilities, according to eqs. (2.29)-(2.31) the portal and hidden sector mass matrices are given by (in the case of only one triplet flavon)

$$
M_{D}=M_{31}=\sqrt{3} D_{u} U_{\omega} D_{h}, \quad M_{S}=M_{11} .
$$

Since $16_{F}$ and $S$ are in different representations of $A_{4}$, the corresponding mass matrices have different flavor structures and it becomes non-trivial to get certain correlations between them.

\subsection{Scheme with $h_{10}=0$}

Vanishing $h_{10}$ can be achieved, e.g. due to $Z_{2}$ symmetry, under which only $\xi$ and $\overline{16}_{H}$ are odd. Only singlet flavons contribute to the visible sector masses and therefore $m_{D}=$ $m_{D}^{0} \mathbb{1}+M_{33}^{\varphi}$ with elements fixed by masses of charged leptons (or up-type quarks), i.e. $m_{D}=m_{l}=m_{l}^{\text {diag }}$. So, mixing is generated by neutrinos. The light neutrino mass matrix can be written as

$$
m_{\nu}=m_{l}^{\operatorname{diag}}\left(M_{31}^{T}\right)^{-1} M_{S}\left(M_{31}\right)^{-1} m_{l}^{\operatorname{diag}},
$$

or explicitly, according to eq. (4.2)

$$
m_{\nu}=\frac{1}{3} \frac{m_{l}^{\text {diag }}}{D_{u}} U_{\omega}^{*} \frac{1}{D_{h}} M_{S} \frac{1}{D_{h}} U_{\omega}^{*} \frac{m_{l}^{\text {diag }}}{D_{u}} .
$$

For $D_{h} \propto \mathbb{1}$ and $m_{l}^{\text {diag }}\left(D_{u}\right)^{-1} \propto \mathbb{1}$ (partial screening), the expression (4.3) is reduced to

$$
m_{\nu} \propto \frac{1}{3} U_{\omega}^{*} M_{S} U_{\omega}^{*}
$$

If the bare mass term dominates in $M_{S}$ we would get

$$
m_{\nu}=\frac{1}{9}\left[3 \tilde{M}_{S}^{0} \mathbb{1}+\left(M_{S}^{0}-\tilde{M}_{S}^{0}\right) M_{\mathrm{dem}}\right]
$$

where $M_{\text {dem }}$ is the matrix with all the elements equal 1 . It can be diagonalized by the TBM mixing but has two equal neutrino masses. Such a degeneracy means that the mixing is not 


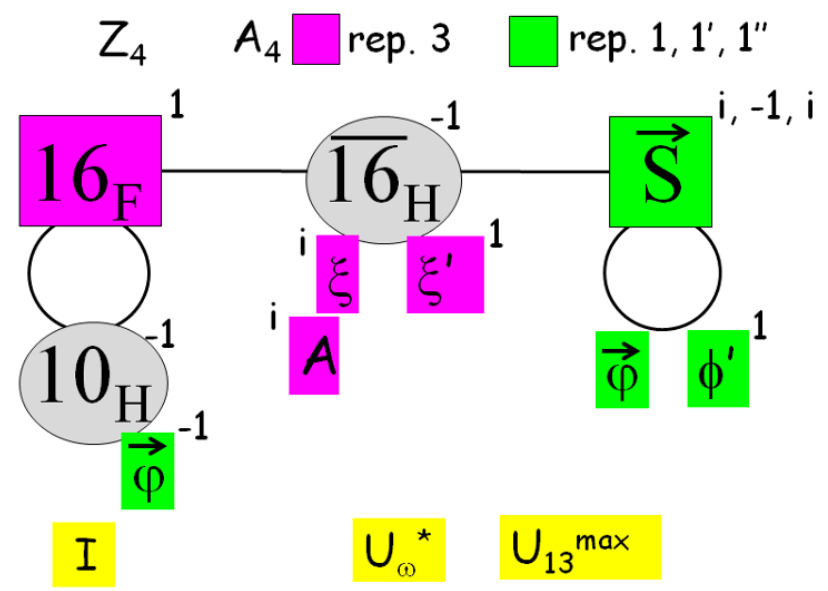

Figure 7. The same as in figure 1 but for the flavon scheme with $S$ in three singlet representations of $A_{4}$ and $h_{10}=0$.

fixed completely. More contributions are required to break it. A straightforward possibility is to use additional contributions to $M_{11}$ in order to obtain maximal 1-3 mixing from $M_{S}$, so that $U_{0}=U_{\omega} U_{13}^{\max }=U_{\mathrm{TBM}}$.

In what follows we will consider another way of getting the required corrections, which also justifies some assumptions we have made before. We elaborate on a scenario which realizes relations

$$
m_{D}=\operatorname{diag}, \quad m_{D}^{T} M_{D}^{-1} \propto U_{\omega}^{*}, \quad D_{h}^{-1} M_{S} D_{h}^{-1}=M_{13}^{\max } .
$$

Here, as in the previous case, the first relation means that $U_{l}=\mathbb{1}$, the second relation corresponds to partial screening of the Dirac structures which generates the magic matrix. The singlet matrix together with $D_{h}$ produces maximal 1-3 mixing. Then $U_{0} \propto U_{\omega} U_{13}^{\max }=U_{\mathrm{TBM}}$.

We introduce $Z_{4}$ to forbid $h_{10}$ and some other couplings. The field content, symmetry assignments and graphic representation of the scheme are given in figure 7 . The features of the scheme (as compared with minimal structure discussed in the beginning of the subsection) are (i) different $Z_{4}$ charges of fermion singlets, (ii) two flavon triplets participating in the portal interactions, (iii) additional flavon singlet $\phi^{\prime}$, (iv) auxiliary flavon triplet $A$.

The most general expressions for the three terms of the Lagrangian (2.1) allowed by symmetry in the lowest order are

$$
\begin{aligned}
\mathcal{L}_{D} & =\frac{10_{H}}{\Lambda_{f}} \vec{y}_{10}\left(16_{F} \cdot 16_{F}\right)_{\varphi} \vec{\varphi}, \\
\mathcal{L}_{N S} & =\frac{\overline{16}_{H}}{\Lambda_{f}}\left[h_{16}\left(16_{F} \cdot \xi\right) S+h_{16}^{\prime}\left(16_{F} \cdot \xi^{\prime}\right)^{\prime \prime} S^{\prime}+h_{16}^{\prime \prime}\left(16_{F} \cdot \xi\right)^{\prime} S^{\prime \prime}\right], \\
\mathcal{L}_{S} & =y_{1}(S \cdot S) \varphi+2 y_{1}^{\prime}\left(S \cdot S^{\prime \prime}\right) \varphi^{\prime}+y_{1}^{\prime \prime}\left(S^{\prime \prime} \cdot S^{\prime \prime}\right) \varphi^{\prime \prime}+\tilde{y}_{1}^{\prime}\left(S^{\prime} \cdot S^{\prime}\right) \phi^{\prime} .
\end{aligned}
$$

Notice that $Z_{4}$ forbids the low-dimension flavonless term $16_{F} 16_{F} 10_{H}$ since $10_{H}$ has the charge -1 . It also ensures that triplet flavons do not couple with $16_{F} 16_{F}$, and therefore 
only singlets contribute to the Dirac mass matrix, leading to $m_{D}=M_{33}^{\varphi}$. The symmetry $Z_{4}$ also forbids the bare mass terms $(S S)$ and $\left(S^{\prime} S^{\prime \prime}\right)$.

There is a generic problem here: on the one hand, obtaining $U_{\omega}$ from $M_{D}$ requires (in the simplest case) that only one flavon triplet participates in the portal interactions. On the other hand, to get maximal 1-3 mixing from the hidden sector one needs to distinguish $S^{\prime}$ from $S$ and $S^{\prime \prime}$, so that the terms $\left(S S^{\prime}\right) \varphi^{\prime \prime}$ and $\left(S^{\prime} S^{\prime \prime}\right) \varphi$ are forbidden. To forbid such terms, singlets $S$ and $S^{\prime \prime}$ should have equal $Z_{4}$ charges which differ from the charge of $S^{\prime}$. Therefore in $\mathcal{L}_{N S}$ two flavon triplets $\xi$ and $\xi^{\prime}$ carrying different $Z_{4}$ charges are necessary. Moreover, a new flavon singlet, $\phi^{\prime}$, with $Z_{4}$ charge different from $\vec{\varphi}$, should be introduced to generate the mass of $S^{\prime}$.

Now in the visible sector, both the bare mass and off-diagonal elements of $M_{33}$ are absent, so $\mathcal{L}_{D}$ in eq. (4.4) only generates the same diagonal Dirac mass matrices $m_{D}=$ $M_{33}^{\varphi}\left(\vec{y}_{10}, \vec{v}\right)$ to all SM fermion components as in eq. (2.20)

$$
\left(m_{D 1}, m_{D 2}, m_{D 3}\right)^{T}=\frac{\left\langle 10_{H}\right\rangle}{\Lambda_{f}} \sqrt{3} U_{\omega}\left(y_{10} v, y_{10}^{\prime} v^{\prime}, y_{10}^{\prime \prime} v^{\prime \prime}\right)^{T}
$$

So the expressions of mass matrices are in the flavor basis from the beginning. ${ }^{5}$ The hierarchy of mass eigenvalues $m_{D 1}, m_{D 2}, m_{D 3}$ can be obtained in the same way as eq. (2.22).

The portal mass matrix generated by the terms $\mathcal{L}_{N S}$ equals

$$
M_{D}=\frac{\left\langle\overline{16}_{H}\right\rangle}{\Lambda_{f}}\left(\begin{array}{ccc}
h_{16} u_{1} & h_{16}^{\prime} u_{1}^{\prime} & h_{16}^{\prime \prime} u_{1} \\
h_{16} u_{2} & h_{16}^{\prime} u_{2}^{\prime} \omega & h_{16}^{\prime \prime} u_{2} \omega^{2} \\
h_{16} u_{3} & h_{16}^{\prime} u_{3}^{\prime} \omega^{2} & h_{16}^{\prime \prime} u_{3} \omega
\end{array}\right) \text {, }
$$

where the VEVs of two flavon triplet are denoted as $\langle\xi\rangle=\left(u_{1}, u_{2}, u_{3}\right),\left\langle\xi^{\prime}\right\rangle=\left(u_{1}^{\prime}, u_{2}^{\prime}, u_{3}^{\prime}\right)$. To get exactly the form of $M_{31}$, their VEVs need to be aligned in the same way: $\langle\xi\rangle=\left\langle\xi^{\prime}\right\rangle$ (i.e. $u_{i}=u_{i}^{\prime}$ ). With this equality, the mass matrix of eq. (4.6) can be written as in eq. (2.29): $M_{D}=\sqrt{3}\left\langle\overline{16}_{H}\right\rangle D_{u} U_{\omega} D_{h} / \Lambda_{f}$.

The mass matrix of singlet fermions generated by eq. (4.4) has the form of $M_{11}$ with zeros due to symmetry assignment:

$$
M_{S}=\left(\begin{array}{ccc}
y_{1} v & 0 & y_{1}^{\prime} v^{\prime} \\
\ldots & \tilde{y}_{1}^{\prime} v_{1} & 0 \\
\ldots & \ldots & y_{1}^{\prime \prime} v^{\prime \prime}
\end{array}\right)
$$

where $v_{1} \equiv\left\langle\phi^{\prime}\right\rangle$.

Combining $M_{D}$ and $M_{S}$ we obtain from eq. (2.35) the light neutrino mass matrix

$$
m_{\nu}=\frac{1}{3}\left(\frac{\Lambda_{f}}{\left\langle\overline{16}_{H}\right\rangle}\right)^{2} \frac{m_{D}^{\mathrm{diag}}}{D_{u}} \cdot U_{\omega}^{*} \cdot\left(\begin{array}{ccc}
\frac{y_{1} v}{h_{16}^{2}} & 0 & \frac{y_{1}^{\prime} v^{\prime}}{h_{16} h_{16}^{\prime \prime}} \\
\ldots & \frac{\tilde{y}_{1}^{\prime} v_{1}}{h_{16}^{\prime 2}} & 0 \\
\ldots & \ldots & \frac{y_{1}^{\prime \prime} v^{\prime \prime}}{h_{16}^{\prime 2}}
\end{array}\right) \cdot U_{\omega}^{*} \cdot \frac{m_{D}^{\mathrm{diag}}}{D_{u}} .
$$

\footnotetext{
${ }^{5}$ The diagonal matrix $m_{D}$ can also be written in the form of product of matrices as $\frac{\left\langle 10_{H}\right\rangle}{\Lambda_{f}} U_{\omega}^{*}\left(y_{10} v \mathbb{1}+\right.$ $\left.y_{10}^{\prime} v^{\prime} C+y_{10}^{\prime \prime} v^{\prime \prime} C^{T}\right) U_{\omega}$, where $C$ is the cyclic matrix with only non-zero $(1,3),(2,1)$, and $(3,2)$ unit elements.
} 
To get the TBM mixing the following two conditions should be fulfilled:

$$
m_{D}^{\operatorname{diag}} \propto D_{u}
$$

which leads to partial screening, and

$$
\frac{y_{1}}{h_{16}^{2}} v=\frac{y_{1}^{\prime \prime}}{h_{16}^{\prime 2}} v^{\prime \prime}
$$

to get maximal 1-3 mixing from the central matrix of eq. (4.8).

The first condition (4.9) is the correlation of the singlet and triplet VEVs, which can be obtained in the same way as it was done in eq. (2.40), by introducing the auxiliary $A_{4}$ triplet field $A$ with VEVs $\langle A\rangle=(a, a, a) / \sqrt{3}$. The inequality $m_{D} \ll M_{D}$ implies a very small VEV: $a \ll 1$. Therefore the auxiliary field does not change other structures of the model. Provided that $y_{10}=y_{10}^{\prime}=y_{10}^{\prime \prime}$, the Dirac masses can be rewritten as

$$
\left(m_{D 1}, m_{D 2}, m_{D 3}\right)=\frac{\sqrt{3} a y_{10}\left\langle 10_{H}\right\rangle}{\Lambda_{f}}\left(u_{1}, u_{2}, u_{3}\right) .
$$

The second condition, eq. (4.10) can be satisfied if, e.g.

$$
h_{16}=\omega h_{16}^{\prime \prime}, \quad y_{1}=\omega y_{1}^{\prime \prime}, \quad v=\omega v^{\prime \prime} .
$$

These equalities then produce the masses of light neutrinos as

$$
m_{1,3}=C \frac{1}{h_{16}}\left(\frac{y_{1}}{h_{16}} \pm \frac{y_{1}^{\prime}}{h_{16}^{\prime \prime}}\right) v, \quad m_{2}=C \frac{\tilde{y}_{1}^{\prime}}{h_{16}^{\prime 2}} v_{1}, \quad C \equiv \frac{a^{2}\left\langle 10_{H}\right\rangle^{2} y_{10}^{2}}{\left\langle\overline{16}_{H}\right\rangle^{2}} .
$$

Strong normal (inverted) mass hierarchy requires $y_{1} / h_{16} \approx-y_{1}^{\prime} / h_{16}^{\prime \prime}\left(y_{1} / h_{16} \approx y_{1}^{\prime} / h_{16}^{\prime \prime}\right)$.

There exist different ways to avoid the introduction of the second triplet and tuning of its VEVs. One possibility is to use single $\xi$ with $Z_{4}$ charge $i$ and assign to $S^{\prime}$ the same charge $i$ as other singlets have. Then to get nearly maximal 1-3 mixing from the hidden sector additional symmetry, e.g. $Z_{2}$, can be introduced and made conserved in the hidden sector only (see also discussion in section 2.5). If $S^{\prime}$ is odd and other particles of the hidden sector are even, then $M_{S}$ will have the form (4.7) with zero 12- and 23- elements. This additional symmetry is broken in the visible and portal sectors, which have substantially lower energy scale $M_{\mathrm{GUT}}$. So, one expects that corrections to the $Z_{2}$ symmetric results are of the order $M_{\mathrm{GUT}} / M_{\mathrm{Pl}}$, which are not important.

Instead of second flavon triplet $\xi^{\prime}$, second $16_{H}^{\prime}$ with $Z_{4}$ charge $i$ can be introduced in the portal interaction

$$
\frac{h_{16}^{\prime}}{\Lambda_{f}}\left(16_{F} \xi\right)^{\prime \prime} 16_{H}^{\prime} S^{\prime}
$$

The VEVs of 16-plets should be equal, $\left\langle 16_{H}^{\prime}\right\rangle=\left\langle 16_{H}\right\rangle$, to get the matrix factor $U_{\omega}$. Alternatively, to replace $\xi^{\prime}$, one can also introduce a flavon singlet $\phi$ with $Z_{4}$ charge $-i$, which leads to a dimension-6 portal interaction $\left(16_{F} \xi\right)^{\prime \prime} 16_{H} \phi S^{\prime}$.

Notice that this scheme can be made more logical if three $\overline{16}_{H}$ (or three $\xi$ ) with different $Z_{4}$ charges are introduced. 


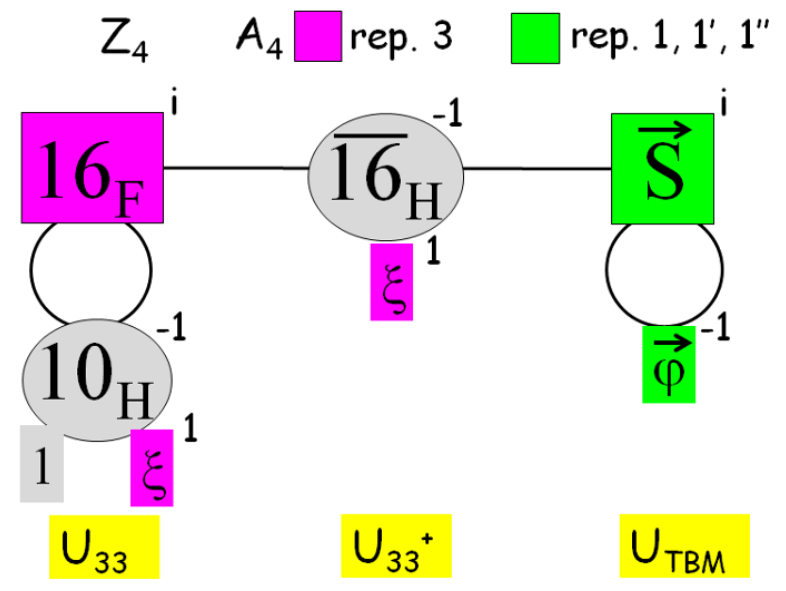

Figure 8. The same as in figure 1 but for the flavon scheme with $S$ in three singlet representations of $A_{4}$ and $\vec{y}_{10}=0$.

\subsection{Scheme with $\vec{y}_{10}=0$}

The visible part of the Lagrangian is as in eq. (3.1) with $\vec{y}_{10}=0$ and $h_{10} \neq 0$. The particle content and symmetry alignment are shown in figure 8. The triplet flavon contributes to both visible and portal interactions, but structures of $m_{D}$ and $M_{D}$ are different. Vanishing $\vec{y}_{10}$ can be obtained with $Z_{4}$ assignment

$$
\left\{16_{F}, S\left|10_{H}, \overline{16}_{H}\right| \vec{\varphi}, \xi\right\} \sim\{i, i|-1,-1|-1,1\},
$$

as in the model of section 3.1. Flavor invariant mass $m_{D}^{0}$ is allowed. Consequently, $m_{D}=$ $m_{D}^{0} \mathbb{1}+M_{33}^{\xi}$, as in eq. (3.6) and $m_{l}=m_{D}$. It is thus diagonalized by $U_{33}$. The portal mass matrix $M_{D}$ has the form of $M_{31}$.

The neutrino mass matrix equals

$$
m_{\nu}=\frac{1}{3} m_{D} \frac{1}{D_{u}} U_{\omega}^{*} \frac{1}{D_{h}} M_{S} \frac{1}{D_{h}} U_{\omega}^{*} \frac{1}{D_{u}} m_{D}
$$

and it converts to

$$
m_{\nu}=\frac{1}{3} m_{D}^{\mathrm{diag}} \cdot U_{33} \frac{1}{D_{u}} U_{\omega}^{*} \cdot \frac{1}{D_{h}} M_{S} \frac{1}{D_{h}} \cdot U_{\omega}^{*} \frac{1}{D_{u}} U_{33} \cdot m_{D}^{\mathrm{diag}}
$$

in the flavor basis. In the case of two Higgs 10-plets, there is actually $m_{D}^{\text {diag }}=m_{u}$. In the hidden sector the bare mass terms, $M_{S}^{0}$ and $\tilde{M}_{S}^{0}$, are forbidden.

If $D_{u}=\mathbb{1}$, the matrix $M_{33}$ has special form $M_{33}^{\text {special }}$, which can be diagonalized by $U_{33}=U_{\omega}$. In this case eq. (4.12) gives

$$
m_{\nu} \propto \frac{1}{3} \frac{m_{D}^{\text {diag }}}{D_{h}} M_{S} \frac{m_{D}^{\text {diag }}}{D_{h}} .
$$

Then for partial screening, $m_{D}^{\text {diag }}\left(D_{h}\right)^{-1} \propto \mathbb{1}$, we obtain $m_{\nu} \propto M_{S}$. With these conditions we essentially reproduce complete screening. Then $M_{S}$ should have the TBM form, 
which can be obtained in the way described by eqs. (2.31), (2.32) in section 2.4. Both normal and inverted neutrino mass hierarchies are possible depending on the choice of the couplings and VEVs.

\section{Generalizations}

We have assumed above that the light neutrino masses are mainly determined by the double seesaw mechanism with three fermion singlets $S$. Here we consider other possible contributions to masses and mixing, including the CKM physics effects.

\subsection{Effects of linear seesaw}

The direct mixing between left-handed neutrinos and fermion singlets $S$ via the $\overline{16}_{H}$ Higgs gives the linear seesaw contribution to the light neutrino masses:

$$
m_{\nu}^{l s}=-m_{D} \frac{1}{M_{D}^{T}} m^{\prime T}-m^{\prime} \frac{1}{M_{D}} m_{D}^{T}
$$

which are the last terms of eq. (2.8). Under the condition $M_{S} \gg M_{\mathrm{cr}}$, where $M_{\mathrm{cr}}$ is defined by eq. (2.10), this contribution is subleading, but may provide certain required corrections to some observables.

On the contrary, if $M_{S} \ll M_{\text {cr }}$, the linear seesaw contribution is dominant. As we have mentioned in section 2.2 in the presence of only one $\overline{16}_{H}, m^{\prime} \propto M_{D}$, and therefore $m_{\nu}^{l s} \propto m_{D}$. Thus, no mixing is generated. If more $\overline{16}_{H}$ multiplets are introduced, the proportionality can be broken: one $\overline{16}_{H}$ with its VEV along the $\mathrm{SU}(2)_{L}$ singlet direction, $\left\langle\overline{16}_{H}\right\rangle_{1}$, will be responsible for $M_{D}$ and another one, $\overline{16}_{H}^{\prime}$, with its VEV along the SU $(2)_{L}$ doublet direction, $\left\langle\overline{16}_{H}\right\rangle_{d}$, generates the mass $m^{\prime}$. If couplings of two 16-plets are different, $y_{16} \neq y_{16}^{\prime}$, the matrices $m^{\prime}$ and $M_{D}$ have different structures, and non-vanishing neutrino mixing can be generated. Notice that for the linear seesaw $M_{S}$ is irrelevant and $m^{\prime}$ plays the role of $M_{S}$.

As an example, we will briefly describe the case when the linear seesaw gives the main contribution to neutrino mass. We assume that $16_{F}$ and $S$ are $A_{4}$ triplets. Then $m_{l}=m_{D}$ can have the form of $M_{\omega}$, as eq. (2.33), which is diagonalized by $U_{\omega}$. Also complete screening can be realized, $m_{D} M_{D}^{-1 T} \propto \mathbb{1}$. For $m^{\prime}$ we can have

$$
m^{\prime}=M_{0} \mathbb{1}+M_{33}^{\xi}
$$

For certain VEV alignment as in the scheme of section 3.5, $\mathrm{m}^{\prime}$ has the form of eq. (2.37), producing the maximal 1-3 rotation, so that $U_{0}=U_{\omega} U_{13}^{\max }=U_{\mathrm{TBM}}$.

Realization of partial screening is different from the case of double seesaw. Let us take a scheme with $16_{F} \sim \mathbf{3}$ and $\vec{S} \sim\left(\mathbf{1}, \mathbf{1}^{\prime}, \mathbf{1}^{\prime \prime}\right)$, in which $m_{D}$ is diagonal, $m_{D} M_{D}^{-1 T} \propto U_{\omega}^{*}$, and $m^{\prime}$ has the form of $M_{31}$. Then the neutrino mass matrix is given by

$$
m_{\nu}^{l s} \propto U_{\omega}^{*} m^{\prime T}+m^{\prime} U_{\omega}^{*} \propto U_{\omega}^{*} D_{h} U_{\omega} D_{u}+D_{u} U_{\omega} D_{h} U_{\omega}^{*}
$$


Under conditions $u_{3}=u_{2}$ in $D_{u}$ and $h_{3}=h_{2}$ in $D_{h}$, which imply the 2-3 permutation symmetry, this matrix gives

$$
m_{\nu}^{l s} \propto\left(\begin{array}{ccc}
2 u_{1}\left(h_{1}+2 h_{2}\right) & \left(u_{1}+u_{2}\right)\left(h_{1}-h_{2}\right) & \left(u_{1}+u_{2}\right)\left(h_{1}-h_{2}\right) \\
\ldots & 2 u_{2}\left(h_{1}+2 h_{2}\right) & 2 u_{2}\left(h_{1}-h_{2}\right) \\
\ldots & \ldots & 2 u_{2}\left(h_{1}+2 h_{2}\right)
\end{array}\right),
$$

with maximal 2-3 and zero 1-3 mixings. Additional assumption $h_{2}=-h_{1}$ is then required to produce the TBM mixing, since $U_{l}=\mathbb{1}$. Three parameters $h_{1}, u_{1}$ and $u_{2}$ allow to get arbitrary masses for the three light neutrinos.

\subsection{Extended hidden sector}

Introduction of more than three fermionic singlets of $\mathrm{SO}(10)$ opens up new possibilities to obtain the observed mixing and masses too. And in some cases it allows to simplify the conditions on couplings and VEVs we imposed in the previous sections. On the other hand, the Dirac screening may not be straightforward any more.

Let us consider for definiteness effects of three additional $\mathrm{SO}(10)$ singlets, so that the hidden fermion sector consists of $S \sim(\mathbf{3}, \overrightarrow{\mathbf{1}})$ of $A_{4}$. The light neutrino mass matrix is given by

$$
m_{\nu}=\left(m_{D}\right)_{3 \times 3} \cdot \frac{1}{\left(M_{D}\right)_{3 \times n} \cdot\left(m_{S}^{-1}\right)_{n \times n} \cdot\left(M_{D}^{T}\right)_{n \times 3}} \cdot\left(m_{D}^{T}\right)_{3 \times 3},
$$

where $n=6$. Taking into account the $A_{4}$ symmetry assignment we can write different mass matrices involved as

$$
m_{D}=M_{33}^{(D)}, \quad M_{D}=\left(M_{33}^{(P)}, M_{31}^{(P)}\right), \quad M_{S}=\left(\begin{array}{cc}
M_{33}^{(S)} & M_{31}^{(S)} \\
M_{13}^{(S)} & M_{11}^{(S)}
\end{array}\right) .
$$

Consider diagonalization of the inverse matrix $\left[m_{\nu}\right]^{-1}$. Recall that if $m_{\nu}$ is diagonalized by the unitary matrix $U$, then $\left[m_{\nu}\right]^{-1}$ is diagonalized by $U^{*}$. Consequently, for a real $U$ as in the case of $U_{\mathrm{TBM}},\left[m_{\nu}\right]^{-1}$ is diagonalized by the same matrix $U$. The inverted mass matrix can be written as

$$
m_{\nu}^{-1}=m_{D}^{-1} M_{N} m_{D}^{-1},
$$

where $M_{N}$ is the effective mass matrix of $\mathrm{RH}$ neutrinos:

$$
M_{N}=M_{D}^{T} M_{S}^{-1} M_{D}
$$

Using expressions (5.2) we find in terms of $3 \times 3$ matrices:

$$
M_{S}^{-1}=\left(\begin{array}{l}
{\left[M_{33}-M_{31} M_{11}^{-1} M_{13}\right]_{S}^{-1}\left[M_{13}-M_{11} M_{31}^{-1} M_{33}\right]_{S}^{-1}} \\
{\left[M_{31}-M_{33} M_{13}^{-1} M_{11}\right]_{S}^{-1}\left[M_{11}-M_{13} M_{33}^{-1} M_{31}\right]_{S}^{-1}}
\end{array}\right),
$$

where the subscript $S$ means that all the matrices in the bracket belong to the hidden sector. Inserting it into eq. (5.4) and then into eq. (5.3) we obtain

$$
\begin{aligned}
m_{\nu}^{-1}= & \left(M_{33}^{(D)}\right)^{-1}\left\{M_{33}^{(P)}\left[M_{33}-M_{31} M_{11}^{-1} M_{13}\right]_{S}^{-1} M_{33}^{(P)}\right. \\
& +M_{33}^{(P)}\left[M_{13}-M_{11} M_{31}^{-1} M_{33}\right]_{S}^{-1} M_{31}^{(P) T}+M_{31}^{(P)}\left[M_{31}-M_{33} M_{13}^{-1} M_{11}\right]_{S}^{-1} M_{33}^{(P)} \\
& \left.+M_{31}^{(P)}\left[M_{11}-M_{13} M_{33}^{-1} M_{31}\right]_{S}^{-1} M_{31}^{(P) T}\right\}\left(M_{33}^{(D)}\right)^{-1} .
\end{aligned}
$$


Imposing various conditions on the matrices involved, one can get specific structures of $m_{\nu}^{-1}$ and lepton mixing.

Let us consider a special case of $M_{13}^{(S)} \equiv\left(M_{31}^{(S)}\right)^{T}=0$, for which, in fact, we can not use general formula (5.6) with the inverse of $M_{13}^{(S)}$. In this case $M_{33}^{(S)}$ and $M_{11}^{(S)}$ contribute independently to the mass matrix of the RH neutrinos, i.e. the denominator of eq. (5.1), through their own portal interactions. Plugging matrices (5.2) into eq. (5.1) we obtain

$$
m_{\nu}=M_{33}^{(D)} \frac{1}{M_{33}^{(P)}\left[M_{33}^{(S)}\right]^{-1} M_{33}^{(P)}+M_{31}^{(P)}\left[M_{11}^{(S)}\right]^{-1} M_{31}^{(P) T}} M_{33}^{(D)},
$$

whose inverse can be written as

$$
\left[m_{\nu}\right]^{-1}=\left[M_{33}^{(D)}\right]^{-1} M_{33}^{(P)}\left[M_{33}^{(S)}\right]^{-1} M_{33}^{(P)}\left[M_{33}^{(D)}\right]^{-1}+\left[M_{33}^{(D)}\right]^{-1} M_{31}^{(P)}\left[M_{11}^{(S)}\right]^{-1} M_{31}^{(P) T}\left[M_{33}^{(D)}\right]^{-1} .
$$

In the first term the complete screening can be realized (as in the scheme of section 3.2) by

$$
\left[M_{33}^{(D)}\right]^{-1} M_{33}^{(P)} \propto \mathbb{1},
$$

if both matrices are generated by the same triplet $\xi$ only. Furthermore, for the flavon triplet, its VEVs $\langle\xi\rangle=u(1,1,1)$, the singlet matrix in hidden sector $M_{33}^{(S)}=M_{33}^{\text {special }}$. Therefore the first term in eq. (5.8) reduces to $\left[M_{33}^{\text {special }}\right]^{-1}$. It is straightforward to see that the inverse of $M_{33}^{\text {special }}$ has the same special form and therefore can be diagonalized by $U_{\mathrm{TBM}}$.

In the second term of eq. (5.8) the partial screening can be realized as in the scheme of eq. (4.8): $\left[M_{33}^{(D)}\right]\left[M_{31}^{(P)}\right]^{-1} \propto U_{\omega}^{*}$ or $\left[M_{33}^{(D)}\right]^{-1} M_{31}^{(P)} \propto U_{\omega}$. Thus, expression (5.8) reduces to

$$
\left[m_{\nu}\right]^{-1}=\left[M_{33}^{\text {special }}\right]^{-1}+g U_{\omega}\left[M_{11}^{(S)}\right]^{-1} U_{\omega}
$$

where $g$ is a coefficient, which takes care of the proportionality and normalization of matrices, thus it is irrelevant for this discussion.

Finally, the proportionality $M_{11}^{(S)} \propto \mathbb{1}$ can be imposed by certain symmetries that only apply to hidden sector interactions. In this case the second term in eq. (5.9) becomes $g U_{\omega} \mathbb{1} U_{\omega}$, which reproduces exactly the correction $\Delta M$ as in eq. (2.26). This correction removes ambiguity in diagonalization of the first term and fixes the TBM mixing. There are enough parameters left to reproduce the observed neutrino masses.

\subsection{On the CKM new physics}

So far we have discussed situations that no CKM mixing is generated and all the Dirac masses of SM fermions are the same: $m_{u}=m_{d}=m_{l}$, contradicting observations. Additional physics (new fields, interactions) is therefore needed to explain properties of the quark sector. This additional physics should not destroy the constructions in the neutrino sector. Here we outline how this can be done, and refer to [48] for more details. To be in agreement with observations, three aspects should be addressed.

1. Difference of the up- and down- type fermions mass hierarchies.

There are various ways to obtain different mass hierarchies of the "upper" and "down" fermions (in the SM doublets) and generate the CKM mixing. 
(i) A second 10-plet of Higgses, $10_{H}^{d}$, can be introduced to give masses of the down fermions, whereas the first one, $10_{H}^{u}$, generates masses of the upper fermions: up-type quarks and neutrinos. Eq. (2.2) then results in

$$
m_{u}=m_{D}=Y_{10}^{u} v_{u}, \quad m_{d}=m_{l}=Y_{10}^{d} v_{d}
$$

Therefore, different Yukawa coupling matrices $Y_{10}^{u}$ and $Y_{10}^{d}$ produce different mass hierarchies and also CKM mixing.

To keep the neutrino part unchanged we assume that in the symmetry basis the CKM mixing comes from the down fermions. That is, couplings of $10_{H}^{d}$ further violate $A_{4}$, explicitly or spontaneously.

(ii) Another possibility is to keep the single $10_{H}$ but introduce the dimension-5 operator

$$
\frac{1}{\Lambda}\left(16_{F} 16_{H} 16_{H} 16_{F}\right) .
$$

This operator also breaks the mass degeneracy between up- and down- type quarks [72].

(iii) Vector-like families of fermions $10_{F}, \overline{10}_{F}$ can exist. Their mixing with usual $16_{F}$ break the degeneracy between up-type and down-type masses of quarks. The reason is that $10_{F}$ only contains down-type quarks and lepton doublets, but no up-type quarks. The interactions which provide this mixing can originate from renormalizable operators $10_{F} 16_{F} 16_{H}$.

2. Difference of masses of down quarks and charged leptons. To breaks the mass degeneracy between quarks and leptons one can use non-renormalizable terms with additional Higgs multiplets. One option is to introduce 45-plet Higgs scalars $45_{H}$ and the dimension-5 operator

$$
\frac{1}{\Lambda}\left(16_{F} \cdot 10_{H}^{d} 45_{H} \cdot 16_{F}\right)
$$

with $45_{H}$ and $10_{H}^{d}$ only [74]. The product of $10_{H}^{d}$ and $45_{H}$ has the decomposition $10 \times 45=10+120+320$, which contains $120_{H}$. If $45_{H}$ acquires its VEV in the direction of $(B-L)$, where $B(L)$ is the baryon (lepton) number, then the effective VEV of $120_{H}$ leads via interactions (5.11) to difference of masses of the second fermion generation (e.g. $m_{\mu}$ and $\left.m_{s}\right)$.

If more than one 45-plet exist, the dimension- 6 operators

$$
\frac{1}{\Lambda^{2}}\left(16_{F} \cdot 45_{H}^{a} 10_{H} 45_{H}^{b} \cdot 16_{F}\right)
$$

give contributions to the masses of the first generation of fermions [29, 75].

3. Nearly the same CKM-type mixing of down-type quarks and charged leptons despite the different mass hierarchies of them. In our framework the CKM matrix is generated by $m_{d}$, and the CKM type correction to lepton mixing which lead to expression (1.1) 
are generated by $m_{l}$. Since masses of the down-type quarks and charged leptons are different, the mass matrices $m_{d}$ and $m_{l}$ should be different, and therefore in general, one expects that diagonalization matrices $U_{d}$ and $U_{l}$ that diagonalize them are also different. This may invalidate eq. (1.1), which implies that both matrices should be diagonalized by the same rotation $U_{l} \simeq U_{d}[48]$.

One solution to this problem was described before in section 3.2. The mass matrix can be the sum of a diagonal matrix proportional to the unit matrix and an off-diagonal matrix:

$$
m_{0} \mathbb{1}+M_{33}^{\xi} .
$$

The mixing is determined by $M_{33}^{\xi}$ only, whereas the eigenvalues are given by the total matrix, which depends on $m_{0}$ and $M_{33}^{\xi}$. So, by varying $m_{0}$, we can change the eigenvalues without modifying the mixing.

\section{Conclusion}

1. We have considered the lepton mixing and neutrino masses in the framework of $\mathrm{SO}(10)$ GUTs with the hidden sector composed of fermionic and bosonic singlets of $\mathrm{SO}(10)$, and flavor symmetries realized in both visible and hidden sectors. Two sectors communicate via the portal interactions provided by $\overline{16}_{H}$ of Higgses.

The framework allows to disentangle the CKM new physics and the neutrino new physics. The neutrino new physics explains the smallness of neutrino masses and generates the large lepton mixing. The CKM new physics, being common for quarks and leptons due to GUT, is responsible for the CKM mixing and difference of mass hierarchies of the up-type and down-type quarks, as well as the charged leptons. The framework naturally realizes the relation between the quark and lepton mixings: $U_{\mathrm{PMNS}} \sim V_{\mathrm{CKM}}^{\dagger} U_{0}$, where the structure of matrix $U_{0}$ is determined by the flavor symmetry.

In the lowest-order approximation with neutrino physics only: (i) quark and lepton masses are generated, (ii) no quark mixing appears, (iii) large lepton mixing is produced, whose form is dictated by the flavor symmetry, (iv) small neutrino masses are generated via the double seesaw mechanism, or its generalizations are related to more than three fermionic singlets.

The CKM physics gives corrections (or perturbations) to this lowest-order picture and the corrections are related to further violation of flavor symmetry effects and higher-order operators.

2. We have mainly focussed on the neutrino new physics and generation of $U_{0}$ in this paper. Using $A_{4} \times Z_{4}$ as the simplest example of flavor group, we have systematically explored the flavor structures (masses, mixing) which can be obtained in this framework, depending on the field content and symmetry assignment. The interplay of GUT SO(10) symmetry and flavor symmetries is crucial in obtaining certain flavor structures. 
In this framework three different mass matrices are involved in generation of lepton mixing: $m_{D}$ - the Dirac mass matrix of SM fermions (charged leptons and neutrinos), $M_{D}$ - the portal mass matrix, or $m_{D} M_{D}^{-1 T}$ — the portal factor, and the matrix of fermion singlets $M_{S}$. This leads to new possibilities to explain the observed mixing. One of the key elements, which allows to disentangle the quark masses and mixing from neutrino masses and mixing, is the Dirac screening (either complete or partial).

Generically, even without flavor symmetries, the framework explains no or small quark mixing, large lepton mixing and small neutrino masses. It leads to the relation between the quark and lepton mixings of the form (1.1).

$U_{0}$ in eq. (1.1) can be factorized as $U_{0}=U_{1} U_{2} \ldots$, where $U_{i}$ are the matrices of maximal two-generation rotation or matrices of special type, such as the magic matrix $U_{\omega}$. Then different mass matrices can be responsible for different rotations $U_{i}$. In this connection we studied properties of matrices $m_{D}, M_{D}, M_{S}$ generated by different $\mathrm{SO}(10)$ Higgs multiplets. Depending on $A_{4}$ assignments for fermions they can be of 5 types: $M_{33}^{\text {diag }}, M_{33}^{\xi}, M_{31}, M_{11}$ and $M_{0} \cdot \mathbb{1}$.

For a complete set of flavons (triplet and singlets) with arbitrary couplings and VEVs, any (symmetric) structure of $M_{33}$ and $M_{11}$ (and consequently, $m_{D}$ and $M_{S}$ ) and $M_{31}$ can be obtained. As a consequence, the flavor structure of light neutrino mass matrix is arbitrary.

The matrices acquire certain flavor structures if only specific flavons (e.g. only triplet) contribute to their generation. If the same flavons contribute to both $m_{D}$ and $M_{D}$, the Dirac screening can be obtained. This can be achieved by additional symmetry, e.g. $Z_{4}$. This symmetry forbids certain couplings of flavons, ensuring that different flavons participate in different types of interactions. It can then explains that some flavons appear in the hidden sector only. Or on the contrary, a common symmetry assignment ensures that the same flavons contribute to different mass matrices.

It is also possible that certain symmetries exist in the hidden sector only, being broken (spontaneously or explicitly) by low scale interactions in the visible and portal interactions.

To have a complete determination of masses and mixing, in particular, to obtain $U_{0} \sim U_{\mathrm{TBM}}$, or $U_{\mathrm{BM}}$, further restrictions are needed. We found the following generic conditions, of which all or some should be satisfied in specific schemes:

- equality (or proportionality) of the flavon singlet couplings $\vec{y}$ in the visible sector (i.e. with $10_{H}$ ) and in portal (with $\overline{16}_{H}$ ) interactions;

- equality of flavon singlet VEVs;

- correlation between VEVs of flavon singlets and triplet;

- certain VEV alignment of triplet(s) $\xi$ including equality of three VEVs, or nonzero VEV of only one component of the triplet; 
- corrections to special matrices which break degeneracy of mass eigenstates and thus fix the mixing.

These relations can be manifestations of further unification, additional symmetries, or selection of certain minimum of the potential from several (degenerate) possibilities. In some cases correlations of the bare mass terms or flavonless contributions are also required.

3. We have presented several schemes with minimal possible number of flavons and couplings in the visible and portal sectors. Interesting realizations are when $U_{\mathrm{TBM}}$, $U_{\mathrm{BM}}$, or certain factors of these matrices follow from different sectors:

- the TBM mixing from the hidden sector $M_{S}$ (with complete screening);

- approximate TBM mixing from charged leptons;

- approximate TBM mixing from the portal and hidden sectors;

- the magic matrix from charged leptons and maximal 1-3 mixing from the hidden sector, thus reproducing $U_{\mathrm{TBM}}$;

- the magic matrix from the portal (partial screening) and maximal 1-3 mixing from the hidden sector;

- the 2-3 maximal mixing from charged leptons and the 1-2 maximal mixing from $M_{S}$, thus reproducing $U_{\mathrm{BM}}$.

In many cases explanations of masses and mixing decouple from each other. This is because of the special (maximal, magic) form of the mixing.

We also commented on possible effects of the linear seesaw and additional fermion singlets in the hidden sector. They can provide required corrections to the zero-order structures.

We outlined the CKM physics, indicating additional elements which could be introduced to obtain the quark sector. We showed that they can be introduced in a consistent way without destroying the part related to neutrino new physics.

4. This is an initial study - the first step toward the model building, which shows, nevertheless, interesting potential to explain fermion masses and mixing. In complete models one needs to further introduce the scalar potential explicitly and find its minima, to consider effects of high-order operators, and to take into account renormalization group effects, etc. Other flavor symmetry groups can be studied. All these issues are beyond the scope of the present paper.

5. The key challenge is the possibility of testing this framework experimentally. In some cases (or with additional assumptions) one can obtain certain predictions for the neutrino mass hierarchy, the type of mass spectrum, value of the Dirac CP phase and values of Majorana phases. Recall that in most of the scenarios considered here there exist enough free parameters (couplings, VEVs) to accommodate any neutrino 
mass spectrum and therefore any type of mass hierarchy. As usual, $A_{4}$ symmetry restricts mixing but not masses, and additional restrictions on the field content and some additional symmetries should be introduced to fix the spectrum. Discovery of the proton decay would be a partial confirmation of the GUT framework. The leptogenesis can give some probes of the framework too.

The hidden sector can provide stable particles to act as dark matter candidates. So, detection of dark matter particles may shed some light on the hidden sector, and consequently, the framework studied in this paper. Sterile neutrinos, if exist, can also originate from such a hidden sector.

\section{A $\quad A_{4}$ group}

In the context of $A_{4}$ flavor symmetry, we use decomposition of product of two triplets $a, b$ as follows

$$
\begin{aligned}
\mathbf{1} & =(a b)=a_{1} b_{1}+a_{2} b_{2}+a_{3} b_{3}, \\
\mathbf{1}^{\prime} & =(a b)^{\prime}=a_{1} b_{1}+\omega^{2} a_{2} b_{2}+\omega a_{3} b_{3}, \\
\mathbf{1}^{\prime \prime} & =(a b)^{\prime \prime}=a_{1} b_{1}+\omega a_{2} b_{2}+\omega^{2} a_{3} b_{3}, \\
\mathbf{3} & =a \cdot b=h_{1} \cdot\left(a_{2} b_{3}, a_{3} b_{1}, a_{1} b_{2}\right)+h_{2} \cdot\left(a_{3} b_{2}, a_{1} b_{3}, a_{2} b_{1}\right),
\end{aligned}
$$

where $\omega=e^{2 \pi i / 3}$, and $h_{1}$ and $h_{2}$ are both free number parameters. We further assume that $h_{1}=h_{2}$ is protected by some underlying mechanism, and in practice choose the value of $h_{1}$ to be 1 because it can be absorbed into relevant Yukawa couplings. Generally, this assumption does not affect the forms of $m_{D}$ and $M_{S}$, which are required to be symmetric anyway. But in the case that both $16_{F i}$ and $S_{i}$ are triplets under $A_{4}$, this assumption leads to a symmetric $M_{D}$, and thus helps to preserve the screening condition which links $M_{D}$ to $m_{D}$.

\section{B Eigenvalues of $M_{33}^{\varphi}$}

Let us denote $\mu \equiv m_{0}+y v, \mu^{\prime} \equiv y^{\prime} v^{\prime}$ and $\mu^{\prime \prime} \equiv y^{\prime \prime} v^{\prime \prime}$. The moduli of the eigenstates of $M_{33}^{\varphi}(2.19)$ equal

$$
\begin{aligned}
& \left|M_{1}\right|=\left|\mu+\mu^{\prime}+\mu^{\prime \prime}\right| \\
& \left|M_{2}\right|=\left[|\mu|^{2}+\left|\mu^{\prime}\right|^{2}+\left|\mu^{\prime \prime}\right|^{2}+2 \operatorname{Re}\left(\omega^{2} \alpha\right)\right]^{1 / 2} \\
& \left|M_{3}\right|=\left[|\mu|^{2}+\left|\mu^{\prime}\right|^{2}+\left|\mu^{\prime \prime}\right|^{2}+2 \operatorname{Re}(\omega \alpha)\right]^{1 / 2}
\end{aligned}
$$

and $\alpha \equiv \mu \mu^{\prime *}+\mu^{\prime} \mu^{\prime \prime *}+\mu^{\prime \prime} \mu^{*}$. The arguments of $M_{2}$ and $M_{3}$ are given by

$$
2 \arctan \left[\frac{\operatorname{Im}\left(2 \mu-\mu^{\prime}-\mu^{\prime \prime}\right) \pm \sqrt{3} \operatorname{Re}\left(\mu^{\prime}-\mu^{\prime \prime}\right)}{\left|2 M_{2,3}\right|+\operatorname{Re}\left(2 \mu-\mu^{\prime}-\mu^{\prime \prime}\right) \pm \sqrt{3} \operatorname{Im}\left(\mu^{\prime \prime}-\mu^{\prime}\right)}\right],
$$

respectively. If $\alpha$ is real, the moduli of two eigenstates are degenerate. 


\section{Diagonalization of $M_{33}^{\xi}$}

Consider the diagonalization of the real matrix

$$
M_{33}^{\xi}=\left(\begin{array}{ccc}
0 & u_{3} & u_{2} \\
u_{3} & 0 & u_{1} \\
u_{2} & u_{1} & 0
\end{array}\right) .
$$

Its eigenvalues are given by

$$
\begin{aligned}
& m_{1}=\frac{2}{3} \sqrt{3 u_{1}^{2}+3 u_{2}^{2}+3 u_{3}^{2}} \cdot \cos \frac{\Delta-2 \pi}{3}, \\
& m_{2}=\frac{2}{3} \sqrt{3 u_{1}^{2}+3 u_{2}^{2}+3 u_{3}^{2}} \cdot \cos \frac{\Delta+2 \pi}{3}, \\
& m_{3}=\frac{2}{3} \sqrt{3 u_{1}^{2}+3 u_{2}^{2}+3 u_{3}^{2}} \cdot \cos \frac{\Delta}{3},
\end{aligned}
$$

where

$$
\cos \Delta \equiv \frac{27 u_{1} u_{2} u_{3}}{\left(3 u_{1}^{2}+3 u_{2}^{2}+3 u_{3}^{2}\right)^{3 / 2}} .
$$

If the elements are nearly degenerate as $u_{i}=u+\epsilon_{i}$, where $\epsilon_{i} \ll u$, we have

$$
\begin{aligned}
& m_{1} \simeq-u-\frac{1}{3}\left(\epsilon_{1}+\epsilon_{2}+\epsilon_{2}-2 \sqrt{\epsilon_{1}^{2}+\epsilon_{2}^{2}+\epsilon_{3}^{2}-\epsilon_{1} \epsilon_{2}-\epsilon_{2} \epsilon_{3}-\epsilon_{3} \epsilon_{1}}\right), \\
& m_{2} \simeq-u-\frac{1}{3}\left(\epsilon_{1}+\epsilon_{2}+\epsilon_{2}+2 \sqrt{\epsilon_{1}^{2}+\epsilon_{2}^{2}+\epsilon_{3}^{2}-\epsilon_{1} \epsilon_{2}-\epsilon_{2} \epsilon_{3}-\epsilon_{3} \epsilon_{1}}\right), \\
& m_{3} \simeq 2 u+\frac{2}{3}\left(\epsilon_{1}+\epsilon_{2}+\epsilon_{2}\right) .
\end{aligned}
$$

Under the condition that $\left|u_{3}\right| \leq u_{2} \leq u_{1}$, it is possible to give the three mixing angles [76]:

$$
\begin{aligned}
\cos ^{2} \theta_{13} & =\frac{2 m_{3}^{2}-u_{3}^{2}-u_{2}^{2}}{\left(m_{3}-m_{1}\right)\left(m_{3}-m_{2}\right)}, \\
\sin ^{2} \theta_{12} & =\frac{\left(m_{3}-m_{1}\right) \cos ^{2} \theta_{13}-m_{3}}{\left(m_{2}-m_{1}\right) \cos ^{2} \theta_{13}}, \\
\cos 2 \theta_{23} & =\frac{\left(m_{1}-m_{2}\right) \sin \theta_{13} \sin 2 \theta_{12}}{2 u_{1}} .
\end{aligned}
$$

Note that the mass matrix always can be redefined to satisfy this condition.

\section{The TBM and BM mixings}

The TBM mixing

$$
U_{\mathrm{TBM}}=\left(\begin{array}{ccc}
\sqrt{\frac{2}{3}} & \frac{1}{\sqrt{3}} & 0 \\
-\frac{1}{\sqrt{6}} & \frac{1}{\sqrt{3}} & -\frac{1}{\sqrt{2}} \\
-\frac{1}{\sqrt{6}} & \frac{1}{\sqrt{3}} & \frac{1}{\sqrt{2}}
\end{array}\right),
$$


can be generated by the physical neutrino mass matrix in the flavor basis

$$
m_{\nu}=\left(\begin{array}{ccc}
x+z & y & y \\
y & z & x+y \\
y & x+y & z
\end{array}\right),
$$

whose form is referred to as $M_{\mathrm{TBM}}$ in the main text. Here $x, y, z$ are free parameters. How to obtain TBM in other bases have been investigated in section 2.5 above.

The BM mixing

$$
U_{\mathrm{BM}}=\left(\begin{array}{ccc}
\frac{1}{\sqrt{2}} & -\frac{1}{\sqrt{2}} & 0 \\
\frac{1}{2} & \frac{1}{2} & -\frac{1}{\sqrt{2}} \\
\frac{1}{2} & \frac{1}{2} & \frac{1}{\sqrt{2}}
\end{array}\right),
$$

corresponds to the neutrino mass matrix in the flavor basis

$$
m_{\nu}=\left(\begin{array}{ccc}
x & y & y \\
y & z & x-z \\
y & x-z & z
\end{array}\right) .
$$

Open Access. This article is distributed under the terms of the Creative Commons Attribution License (CC-BY 4.0), which permits any use, distribution and reproduction in any medium, provided the original author(s) and source are credited.

\section{References}

[1] J.C. Pati and A. Salam, Unified Lepton-Hadron Symmetry and a Gauge Theory of the Basic Interactions, Phys. Rev. D 8 (1973) 1240 [InSPIRE].

[2] H. Georgi and S.L. Glashow, Unity of All Elementary Particle Forces, Phys. Rev. Lett. 32 (1974) 438 [INSPIRE].

[3] H. Georgi, The State of the Art - Gauge Theories, AIP Conf. Proc. 23 (1975) 575 [InSPIRE].

[4] H. Fritzsch and P. Minkowski, Unified Interactions of Leptons and Hadrons, Annals Phys. 93 (1975) 193 [INSPIRE].

[5] P. Minkowski, $\mu \rightarrow$ er at a Rate of One Out of $10^{9}$ Muon Decays?, Phys. Lett. B 67 (1977) 421 [INSPIRE].

[6] T. Yanagida, Horizontal Symmetry And Masses Of Neutrinos, Conf. Proc. C 7902131 (1979) 95 [INSPIRE].

[7] M. Gell-Mann, P. Ramond and R. Slansky, Complex Spinors and Unified Theories, Conf. Proc. C 790927 (1979) 315 [arXiv:1306.4669] [INSPIRE].

[8] S.L. Glashow, The Future of Elementary Particle Physics, NATO Sci. Ser. B 61 (1980) 687 [INSPIRE].

[9] R.N. Mohapatra and G. Senjanović, Neutrino Masses and Mixings in Gauge Models with Spontaneous Parity Violation, Phys. Rev. D 23 (1981) 165 [INSPIRE].

[10] R.N. Mohapatra and J.W.F. Valle, Neutrino Mass and Baryon Number Nonconservation in Superstring Models, Phys. Rev. D 34 (1986) 1642 [INSPIRE]. 
[11] A.Yu. Smirnov, Seesaw enhancement of lepton mixing, Phys. Rev. D 48 (1993) 3264 [hep-ph/9304205] [INSPIRE].

[12] A.Yu. Smirnov, Neutrinos and structure of the intermediate mass scale, Nucl. Phys. B 466 (1996) 25 [hep-ph/9511239] [INSPIRE].

[13] M. Tanimoto, Seesaw enhancement of neutrino mixing due to the right-handed phases, Phys. Lett. B 345 (1995) 477 [hep-ph/9503318] [INSPIRE].

[14] P.F. Harrison, D.H. Perkins and W.G. Scott, Tri-bimaximal mixing and the neutrino oscillation data, Phys. Lett. B 530 (2002) 167 [hep-ph/0202074] [INSPIRE].

[15] P.F. Harrison and W.G. Scott, Symmetries and generalizations of tri-bimaximal neutrino mixing, Phys. Lett. B 535 (2002) 163 [hep-ph/0203209] [INSPIRE].

[16] P.F. Harrison and W.G. Scott, Permutation symmetry, tri-bimaximal neutrino mixing and the S3 group characters, Phys. Lett. B 557 (2003) 76 [hep-ph/0302025] [INSPIRE].

[17] P.F. Harrison and W.G. Scott, Status of tri-bimaximal neutrino mixing, hep-ph/0402006 [INSPIRE].

[18] D. Wyler, Discrete Symmetries in the Six Quark SU(2) $\times$ U(1) Model, Phys. Rev. D 19 (1979) 3369 [INSPIRE].

[19] G.C. Branco, H.P. Nilles and V. Rittenberg, Fermion Masses and Hierarchy of Symmetry Breaking, Phys. Rev. D 21 (1980) 3417 [InSPIRE].

[20] E. Ma and G. Rajasekaran, Softly broken $A_{4}$ symmetry for nearly degenerate neutrino masses, Phys. Rev. D 64 (2001) 113012 [hep-ph/0106291] [INSPIRE].

[21] K.S. Babu, E. Ma and J.W.F. Valle, Underlying $A_{4}$ symmetry for the neutrino mass matrix and the quark mixing matrix, Phys. Lett. B 552 (2003) 207 [hep-ph/0206292] [INSPIRE].

[22] M. Hirsch, J.C. Romao, S. Skadhauge, J.W.F. Valle and A. Villanova del Moral, Phenomenological tests of supersymmetric $A_{4}$ family symmetry model of neutrino mass, Phys. Rev. D 69 (2004) 093006 [hep-ph/0312265] [InSPIRE].

[23] G. Altarelli and F. Feruglio, Tri-bimaximal neutrino mixing from discrete symmetry in extra dimensions, Nucl. Phys. B 720 (2005) 64 [hep-ph/0504165] [INSPIRE].

[24] S.F. King and M. Malinsky, $A_{4}$ family symmetry and quark-lepton unification, Phys. Lett. B 645 (2007) 351 [hep-ph/0610250] [INSPIRE].

[25] Y. Koide, $A_{4}$ symmetry and lepton masses and mixing, Eur. Phys. J. C 52 (2007) 617 [hep-ph/0701018] [INSPIRE].

[26] S.M. Barr, Flavor without flavor symmetry, Phys. Rev. D 65 (2002) 096012 [hep-ph/0106241] [INSPIRE].

[27] E. Ma, Quark mass matrices in the $A_{4}$ model, Mod. Phys. Lett. A 17 (2002) 627 [hep-ph/0203238] [INSPIRE].

[28] E. Ma, Suitability of $A_{4}$ as a Family Symmetry in Grand Unification, Mod. Phys. Lett. A 21 (2006) 2931 [hep-ph/0607190] [INSPIRE].

[29] S. Morisi, M. Picariello and E. Torrente-Lujan, Model for fermion masses and lepton mixing in $\mathrm{SO}(10) \times A_{4}$, Phys. Rev. D 75 (2007) 075015 [hep-ph/0702034] [INSPIRE]. 
[30] F. Bazzocchi, S. Morisi, M. Picariello and E. Torrente-Lujan, Embedding $A_{4}$ into $\mathrm{SU}(3) \times \mathrm{U}(1)$ flavor symmetry: Large neutrino mixing and fermion mass hierarchy in $\mathrm{SO}(10)$ GUT, J. Phys. G 36 (2009) 015002 [arXiv:0802.1693] [InSPIRE].

[31] C. Giunti and M. Tanimoto, Deviation of neutrino mixing from bimaximal, Phys. Rev. D 66 (2002) 053013 [hep-ph/0207096] [INSPIRE].

[32] H. Minakata and A.Yu. Smirnov, Neutrino mixing and quark-lepton complementarity, Phys. Rev. D 70 (2004) 073009 [hep-ph/0405088] [INSPIRE].

[33] K. Cheung, S.K. Kang, C.S. Kim and J. Lee, Lepton flavor violation as a probe of quark-lepton unification, Phys. Rev. D 72 (2005) 036003 [hep-ph/0503122] [INSPIRE].

[34] Z.-z. Xing, Nontrivial correlation between the CKM and MNS matrices, Phys. Lett. B 618 (2005) 141 [hep-ph/0503200] [INSPIRE].

[35] A. Datta, L. Everett and P. Ramond, Cabibbo haze in lepton mixing, Phys. Lett. B 620 (2005) 42 [hep-ph/0503222] [INSPIRE].

[36] S. Antusch, S.F. King and R.N. Mohapatra, Quark-lepton complementarity in unified theories, Phys. Lett. B 618 (2005) 150 [hep-ph/0504007] [InSPIRE].

[37] S. Antusch and S.F. King, Charged lepton corrections to neutrino mixing angles and CP phases revisited, Phys. Lett. B 631 (2005) 42 [hep-ph/0508044] [INSPIRE].

[38] J. Harada, Neutrino mixing and CP-violation from Dirac-Majorana bimaximal mixture and quark-lepton unification, Europhys. Lett. 75 (2006) 248 [hep-ph/0512294] [INSPIRE].

[39] Y. Farzan and A.Yu. Smirnov, Leptonic CP-violation: Zero, maximal or between the two extremes, JHEP 01 (2007) 059 [hep-ph/0610337] [INSPIRE].

[40] S. Antusch, C. Gross, V. Maurer and C. Sluka, $\theta^{P} M N S_{13}=\theta_{C} / \sqrt{2}$ from GUTs, Nucl. Phys. B 866 (2013) 255 [arXiv: 1205.1051] [INSPIRE].

[41] S. Antusch, C. Gross, V. Maurer and C. Sluka, A flavour GUT model with $\theta_{13}^{P M N S} \simeq \theta_{C} / \sqrt{2}$, Nucl. Phys. B 877 (2013) 772 [arXiv:1305.6612] [INSPIRE].

[42] S. Antusch, C. Gross, V. Maurer and C. Sluka, Inverse neutrino mass hierarchy in a flavour GUT model, Nucl. Phys. B 879 (2014) 19 [arXiv:1306.3984] [INSPIRE].

[43] F. Vissani, A study of the scenario with nearly degenerate Majorana neutrinos, hep-ph/9708483 [INSPIRE].

[44] M. Fukugita, M. Tanimoto and T. Yanagida, Atmospheric neutrino oscillation and a phenomenological lepton mass matrix, Phys. Rev. D 57 (1998) 4429 [hep-ph/9709388] [INSPIRE].

[45] V.D. Barger, S. Pakvasa, T.J. Weiler and K. Whisnant, Bimaximal mixing of three neutrinos, Phys. Lett. B 437 (1998) 107 [hep-ph/9806387] [INSPIRE].

[46] S. Davidson and S.F. King, Bimaximal neutrino mixing in the MSSM with a single right-handed neutrino, Phys. Lett. B 445 (1998) 191 [hep-ph/9808296] [INSPIRE].

[47] R.N. Mohapatra and S. Nussinov, Bimaximal neutrino mixing and neutrino mass matrix, Phys. Rev. D 60 (1999) 013002 [hep-ph/9809415] [INSPIRE].

[48] P.O. Ludl and A.Yu. Smirnov, Lepton mixing from the hidden sector, Phys. Rev. D 92 (2015) 073010 [arXiv:1507.03494] [INSPIRE]. 
[49] S.F. King, Tri-bimaximal-Cabibbo Mixing, Phys. Lett. B 718 (2012) 136 [arXiv:1205.0506] [INSPIRE].

[50] F. Gursey, P. Ramond and P. Sikivie, A Universal Gauge Theory Model Based on E6, Phys. Lett. B 60 (1976) 177 [inSPIRE].

[51] T.D. Lee and C.-N. Yang, Question of Parity Conservation in Weak Interactions, Phys. Rev. 104 (1956) 254 [INSPIRE].

[52] A. Salam, On parity conservation and neutrino mass, Nuovo Cim. 5 (1957) 299 [INSPIRE].

[53] I.Yu. Kobzarev, L.B. Okun and I.Ya. Pomeranchuk, On the possibility of experimental observation of mirror particles, Sov. J. Nucl. Phys. 3 (1966) 837 [InSPIRE].

[54] M. Lindner, M.A. Schmidt and A.Yu. Smirnov, Screening of Dirac flavor structure in the seesaw and neutrino mixing, JHEP 07 (2005) 048 [hep-ph/0505067] [INSPIRE].

[55] M.A. Schmidt and A.Yu. Smirnov, Quark Lepton Complementarity and Renormalization Group Effects, Phys. Rev. D 74 (2006) 113003 [hep-ph/0607232] [INSPIRE].

[56] C. Hagedorn, M.A. Schmidt and A.Yu. Smirnov, Lepton Mixing and Cancellation of the Dirac Mass Hierarchy in $\mathrm{SO}(10)$ GUTs with Flavor Symmetries T(7) and Sigma(81), Phys. Rev. D 79 (2009) 036002 [arXiv: 0811.2955] [INSPIRE].

[57] P.H. Chankowski and S. Pokorski, Quantum corrections to neutrino masses and mixing angles, Int. J. Mod. Phys. A 17 (2002) 575 [hep-ph/0110249] [InSPIRE].

[58] S.F. King and N.N. Singh, Renormalization group analysis of single right-handed neutrino dominance, Nucl. Phys. B 591 (2000) 3 [hep-ph/0006229] [INSPIRE].

[59] S.F. King and N.N. Singh, Inverted hierarchy models of neutrino masses, Nucl. Phys. B 596 (2001) 81 [hep-ph/0007243] [INSPIRE].

[60] S. Antusch, J. Kersten, M. Lindner and M. Ratz, Neutrino mass matrix running for nondegenerate seesaw scales, Phys. Lett. B 538 (2002) 87 [hep-ph/0203233] [INSPIRE].

[61] B. Stech and Z. Tavartkiladze, Fermion masses and coupling unification in $E_{6}$ : Life in the desert, Phys. Rev. D 70 (2004) 035002 [hep-ph/0311161] [INSPIRE].

[62] A. Zee, A Theory of Lepton Number Violation, Neutrino Majorana Mass and Oscillation, Phys. Lett. B 93 (1980) 389 [Erratum ibid. B 95 (1980) 461] [InSPIRE].

[63] L. Wolfenstein, A Theoretical Pattern for Neutrino Oscillations, Nucl. Phys. B 175 (1980) 93 [INSPIRE].

[64] M. Hirsch, A.S. Joshipura, S. Kaneko and J.W.F. Valle, Predictive flavour symmetries of the neutrino mass matrix, Phys. Rev. Lett. 99 (2007) 151802 [hep-ph/0703046] [INSPIRE].

[65] E. Ma, $A_{4}$ symmetry and neutrinos with very different masses, Phys. Rev. D 70 (2004) 031901 [hep-ph/0404199] [INSPIRE].

[66] E. Ma, Supersymmetric $A_{4} \times Z(3)$ and $A_{4}$ realizations of neutrino tribimaximal mixing without and with corrections, Mod. Phys. Lett. A 22 (2007) 101 [hep-ph/0610342] [INSPIRE].

[67] Y. Lin, A Predictive $A_{4}$ model, Charged Lepton Hierarchy and Tri-bimaximal Sum Rule, Nucl. Phys. B 813 (2009) 91 [arXiv:0804.2867] [INSPIRE].

[68] G. Altarelli and F. Feruglio, Discrete Flavor Symmetries and Models of Neutrino Mixing, Rev. Mod. Phys. 82 (2010) 2701 [arXiv:1002.0211] [inSPIRE]. 
[69] R. Barbieri, L.J. Hall, G.L. Kane and G.G. Ross, Nearly degenerate neutrinos and broken flavor symmetry, hep-ph/9901228 [INSPIRE].

[70] F. Feruglio, C. Hagedorn and L. Merlo, Vacuum Alignment in SUSY A4 Models, JHEP 03 (2010) 084 [arXiv:0910.4058] [INSPIRE].

[71] M. Holthausen and M.A. Schmidt, Natural Vacuum Alignment from Group Theory: The Minimal Case, JHEP 01 (2012) 126 [arXiv:1111.1730] [InSPIRE].

[72] K.S. Babu, J.C. Pati and F. Wilczek, Fermion masses, neutrino oscillations and proton decay in the light of Super-Kamiokande, Nucl. Phys. B 566 (2000) 33 [hep-ph/9812538] [INSPIRE].

[73] G. Altarelli and F. Feruglio, Theoretical models of neutrino masses and mixings, Springer Tracts Mod. Phys. 190 (2003) 169 [hep-ph/0206077] [INSPIRE].

[74] D. Chang, T. Fukuyama, Y.-Y. Keum, T. Kikuchi and N. Okada, Perturbative SO(10) grand unification, Phys. Rev. D 71 (2005) 095002 [hep-ph/0412011] [INSPIRE].

[75] G. Anderson, S. Raby, S. Dimopoulos, L.J. Hall and G.D. Starkman, A systematic SO(10) operator analysis for fermion masses, Phys. Rev. D 49 (1994) 3660 [hep-ph/9308333] [INSPIRE].

[76] M.J. Kronenburg, A Method for Fast Diagonalization of $a 2 \times 2$ or $3 \times 3$ Real Symmetric Matrix, arXiv:1306.6291. 